\title{
BILINEAR WEIGHTED HARDY INEQUALITY FOR NONINCREASING FUNCTIONS
}

\author{
MARTIN KřEPELA
}

\begin{abstract}
We characterize the validity of the bilinear Hardy inequality for nonincreasing functions

$$
\left\|f^{* *} g^{* *}\right\|_{L^{q}(w)} \leq C\|f\|_{\Lambda^{p_{1}\left(v_{1}\right)}}\|g\|_{\Lambda^{p_{2}\left(v_{2}\right)}},
$$

in terms of the weights $v_{1}, v_{2}, w$, covering the complete range of exponents $p_{1}, p_{2}, q \in$ $(0, \infty]$.
\end{abstract}

The problem is solved by reducing it into the iterated Hardy-type inequalities

$$
\begin{aligned}
& \left(\int_{0}^{\infty}\left(\int_{0}^{x}\left(g^{* *}(t)\right)^{\alpha} \varphi(t) \mathrm{d} t\right)^{\frac{\beta}{\alpha}} \psi(x) \mathrm{d} x\right)^{\frac{1}{\beta}} \leq C\left(\int_{0}^{\infty}\left(g^{*}(x)\right)^{\gamma} \omega(x) \mathrm{d} x\right)^{\frac{1}{\gamma}}, \\
& \left(\int_{0}^{\infty}\left(\int_{x}^{\infty}\left(g^{* *}(t)\right)^{\alpha} \varphi(t) \mathrm{d} t\right)^{\frac{\beta}{\alpha}} \psi(x) \mathrm{d} x\right)^{\frac{1}{\beta}} \leq C\left(\int_{0}^{\infty}\left(g^{*}(x)\right)^{\gamma} \omega(x) \mathrm{d} x\right)^{\frac{1}{\gamma}} .
\end{aligned}
$$

Validity of these inequalities is characterized here for $0<\alpha \leq \beta<\infty$ and $0<\gamma<\infty$.

2010 Mathematics Subject Classification: 26D10, 47G10.

Key words: Hardy operators, bilinear operators, weights, inequalities for monotone functions.

\section{Introduction}

Consider the bilinear Hardy operator

$$
H_{2}(f, g)(t):=\frac{1}{t^{2}} \int_{0}^{t} f(s) \mathrm{d} s \int_{0}^{t} g(s) \mathrm{d} s,
$$

defined for all nonnegative measurable functions $f, g$ on $(0, \infty)$. In this article, we will find necessary and sufficient conditions for the boundedness

$$
H_{2}: L_{\mathrm{dec}}^{p_{1}}\left(v_{1}\right) \times L_{\mathrm{dec}}^{p_{2}}\left(v_{2}\right) \rightarrow L^{q}(w)
$$

with $p_{1}, p_{2}, q \in(0, \infty]$. In other words, the goal is to provide equivalent estimates of the constant

$$
C_{(1)}=\sup _{f, g \in \mathscr{M}} \frac{\left\|f^{* *} g^{* *}\right\|_{L^{q}(w)}}{\|f\|_{\Lambda^{p_{1}\left(v_{1}\right)}}\|g\|_{\Lambda^{p_{2}\left(v_{2}\right)}}}
$$

in terms of $p_{1}, p_{2}, q, v_{1}, v_{2}, w$. 
Let us at first summarize the used notation and symbols. Let $(\mathscr{R}, \mu)$ be an arbitrary totally $\sigma$-finite measure space. Then $\mathscr{M}$ denotes the cone of all extended real-valued $\mu$-measurable functions on $\mathscr{R}$. Next, $\mathscr{M}_{+}$denotes the cone of all extended nonnegative Lebesgue-measurable functions on $(0, \infty)$.

If $p \in(0,1) \cup(1, \infty]$, then $p^{\prime}:=\frac{p}{p-1}$. If $p=1$, then $p^{\prime}:=\infty$. Notice that for $p \in(0,1)$ the number $p^{\prime}$ is negative. Furthermore, the conventions " $\frac{0}{0}=0 . \infty:=0$ " and " $\frac{a}{0}:=\infty$ " for $a \in(0, \infty]$ are used throughout the text.

A weight is any nonnegative measurable function $v$ on $(0, \infty)$ such that for all $t \in(0, \infty)$ it holds $0<V(t)<\infty$, where $V$ is defined by $V(t):=$ $\int_{0}^{t} v$. If the weight is denoted by another letter, the corresponding capital letter plays an analogous role.

We say that a function $u \in \mathscr{M}_{+}$is integrable near the origin if there exists $\varepsilon>0$ such that $\int_{0}^{\varepsilon} u<\infty$. Notice that weights are integrable near the origin by definition.

The symbol $A \lesssim B$ means that $A \leq C B$, where $C$ is an absolute constant independent of relevant quantities in $A, B$. In fact, throughout this article such $C$ depends only on the exponents $(p, q, \alpha, \beta$, etc.), thus it does not even depend on the weights. If both $A \lesssim B$ and $B \lesssim A$, we write $A \simeq B$.

By $A_{(\ldots)}$ we denote the characteristic condition which appears on the line denoted by the number in the brackets. Certain significant optimal constants $C_{(\ldots)}$ are denoted in a similar way. These symbols have a unique meaning throughout the whole paper. Symbols $B_{0}, B_{1}$, etc. are used in the proofs as an auxiliary notation for various quantities, and their meaning may differ between the theorems. However, within the proof of a single theorem or lemma, each symbol $B_{i}$ is uniquely defined.

The text deals with various function spaces. The weighted Lebesgue space $L^{p}(v)$ consists of all extended real-valued Lebesgue-measurable functions $h$ on $(0, \infty)$ such that $\|h\|_{L^{p}(v)}<\infty$. The functional $\|\cdot\|_{L^{p}(v)}$ is defined by

$$
\begin{aligned}
\|h\|_{L^{p}(v)}:=\left(\int_{0}^{\infty}|h(x)|^{p} v(x) \mathrm{d} x\right)^{\frac{1}{p}}, & p \in(0, \infty), \\
\left|h \|_{L^{\infty}(v)}:=\operatorname{ess~sup}_{x>0}\right| h(x) \mid v(x), & p=\infty .
\end{aligned}
$$

The symbol $L_{\mathrm{dec}}^{p}(v)$ stands for the set of all nonnegative and nonincreasing functions from $L^{p}(v)$. 
If $f \in \mathscr{M}$, then $f^{*}$ denotes its nonincreasing rearrangement and $f^{* *}$ the Hardy-Littlewood maximal function of $f$, i.e.

$$
f^{* *}(t):=\frac{1}{t} \int_{0}^{t} f^{*}(s) \mathrm{d} s, \quad t>0 .
$$

For details see $[\mathbf{3}]$. For the definitions of rearrangement-invariant (abbreviated r.i.) spaces and r.i. (quasi-)norms see $[\mathbf{3}, \mathbf{7}, \mathbf{1 8}]$. If $X$ and $Y$ are r.i. spaces (or just r.i. lattices), we say that $X$ is embedded into $Y$ and write $X \hookrightarrow Y$ if there exists $C \in(0, \infty)$ such that for all $f \in X$ it holds

$$
\|f\|_{Y} \leq C\|f\|_{X}
$$

The least possible constant $C$ in this inequality is called the optimal constant of the embedding $X \hookrightarrow Y$ and is equal to the norm of the identity operator between $X$ and $Y$, denoted $\|\mathrm{Id}\|_{X \rightarrow Y}$.

Let $v$ be a weight and $p \in(0, \infty]$. The weighted Lorentz spaces $\Lambda^{p}(v)$ and $\Gamma^{p}(v)$ consist of all functions $f \in \mathscr{M}$ for which $\|f\|_{\Lambda^{p}(v)}<\infty$ and $\|f\|_{\Gamma^{p}(v)}<\infty$, respectively. Here it is

$$
\|f\|_{\Lambda^{p}(v)}:=\left\|f^{*}\right\|_{L^{p}(v)} \text { and }\|f\|_{\Gamma^{p}(v)}:=\left\|f^{* *}\right\|_{L^{p}(v)} .
$$

For more information about the Lorentz $\Lambda$ and $\Gamma$ spaces see e.g. [7] and the references therein.

Let $\varphi, \psi$ be weights. For $g \in \mathscr{M}$ define

$$
\begin{aligned}
&\|g\|_{J^{\alpha, \beta}(\varphi, \psi)}:=\left[\int_{0}^{\infty}\left(\int_{0}^{x}\left(g^{* *}(t)\right)^{\alpha} \varphi(t) \mathrm{d} t\right)^{\frac{\beta}{\alpha}} \psi(x) \mathrm{d} x\right]^{\frac{1}{\beta}}, \quad \alpha, \beta \in(0, \infty), \\
&\|g\|_{J^{\alpha, \infty}(\varphi, \psi)}:=\underset{x>0}{\operatorname{ess} \sup }\left(\int_{0}^{x}\left(g^{* *}(t)\right)^{\alpha} \varphi(t) \mathrm{d} t\right)^{\frac{1}{\alpha}} \psi(x), \quad \alpha \in(0, \infty), \\
&\|g\|_{K^{\alpha, \beta}(\varphi, \psi)}:=\left[\int_{0}^{\infty}\left(\int_{x}^{\infty}\left(g^{* *}(t)\right)^{\alpha} \varphi(t) \mathrm{d} t\right)^{\frac{\beta}{\alpha}} \psi(x) \mathrm{d} x\right]^{\frac{1}{\beta}}, \quad \alpha, \beta \in(0, \infty), \\
&\|g\|_{K^{\alpha, \infty}(\varphi, \psi)}:=\operatorname{ess~sup}_{x>0}\left(\int_{x}^{\infty}\left(g^{* *}(t)\right)^{\alpha} \varphi(t) \mathrm{d} t\right)^{\frac{1}{\alpha}} \psi(x), \alpha \in(0, \infty) .
\end{aligned}
$$

Then, as usual, it is $J^{\alpha, \beta}(\varphi, \psi):=\left\{f \in \mathscr{M} ;\|f\|_{J^{\alpha, \beta}(\varphi, \psi)}<\infty\right\}$ and $K^{\alpha, \beta}(\varphi, \psi):=\left\{f \in \mathscr{M} ;\|f\|_{K^{\alpha, \beta}(\varphi, \psi)}<\infty\right\}$. The "K-spaces" were defined in [18], where they appeared as optimal spaces in certain Youngtype convolution inequalities. Besides that, in $[\mathbf{1 6}]$ it was shown that the associate space to the generalized $\Gamma$ space is also a " $K$-space". 
Now, let us briefly present some background to the problems we are about to investigate. The aforementioned operator $\mathrm{H}_{2}$ is a bilinear version of the classical Hardy operator $H_{1}$, which is defined by

$$
H_{1} f(t):=\frac{1}{t} \int_{0}^{t} f(s) \mathrm{d} s
$$

for all $f \in \mathscr{M}_{+}$. Boundedness of $H_{1}$ between weighted Lebesgue spaces is equivalent to the validity of the weighted Hardy inequality

$$
\left[\int_{0}^{\infty}\left(\frac{1}{x} \int_{0}^{x} f(s) \mathrm{d} s\right)^{q} w(x) \mathrm{d} x\right]^{\frac{1}{q}} \leq C\left(\int_{0}^{\infty} f^{p}(x) v(x) \mathrm{d} x\right)^{\frac{1}{p}}
$$

for all $f \in \mathscr{M}_{+}$, with $C$ being a constant independent of $f$. The weights $v, w$ for which this inequality is valid, have been characterized by Muckenhoupt [23], Bradley [5], and Maz'ja [22]. The weighted Hardy inequality has a broad variety of applications and represents now a basic tool in many parts of mathematical analysis, namely in the study of weighted function inequalities. For the results, history, and applications of this problem, see $[\mathbf{2 1}, \mathbf{2 5}, \mathbf{2 0}]$.

In the last decades, much attention has been drawn by the so-called restricted inequalities. By this term it is meant that an inequality is not supposed to be satisfied by the whole set of nonnegative functions, but rather only by a certain, restricted, subset. In this way, one may ask under which conditions the inequality (2) is satisfied for all nonincreasing $f \in \mathscr{M}_{+}$. This is equivalent to the validity of

$$
\left[\int_{0}^{\infty}\left(\frac{1}{t} \int_{0}^{t} f^{*}(s) \mathrm{d} s\right)^{q} w(t) \mathrm{d} t\right]^{\frac{1}{q}} \leq C\left(\int_{0}^{\infty}\left(f^{*}(t)\right)^{p} v(t) \mathrm{d} t\right)^{\frac{1}{p}},
$$

for all $f \in \mathscr{M}$, with an independent $C$. Moreover, this corresponds to the boundedness $H_{1}: L_{\text {dec }}^{p}(v) \rightarrow L^{q}(w)$, or, in yet different words, the existence of the embedding of the Lorentz spaces $\Lambda^{p}(v) \hookrightarrow \Gamma^{q}(w)$.

The first results on the case $\Lambda^{p}(v) \hookrightarrow \Gamma^{p}(v), 1<p<\infty$ were obtained by Boyd [4] and in an explicit form by Ariño and Muckenhoupt [2]. The problem with $v \neq w$ and $p \neq q, 1<p, q<\infty$ was first successfully solved by Sawyer [26]. Many articles on this topic followed, providing the results for a wider range of parameters, see $[\mathbf{3 0 , 8}, \mathbf{9}, \mathbf{2 8}, \mathbf{1 0}, \mathbf{7}, \mathbf{6}]$. In [7] the results available in 2000 were surveyed. 
The restricted operator inequalities may often be handled by the socalled "reduction theorems". These, in general, reduce a restricted inequality into certain nonrestricted inequalities. For example, the restriction to nonincreasing or quasiconcave functions may be handled in this way, see e.g. $[\mathbf{2 7}, \mathbf{1 5}, \mathbf{1 7}, \mathbf{1 2}]$.

Let us however turn the focus to the bilinear variants of the Hardytype inequalities. Recently, Aguilar, Ortega, and Ramírez [1] found necessary and sufficient conditions for the boundedness $H_{2}: L^{p_{1}}\left(v_{1}\right) \times$ $L^{p_{2}}\left(v_{2}\right) \rightarrow L^{q}(\widetilde{w})$, where $\widetilde{w}(t):=t^{2 q} w(t)$. In other words, they characterized the validity of the weighted bilinear Hardy inequality

$$
\left[\int_{0}^{\infty}\left(\int_{0}^{t} f(s) \mathrm{d} s \int_{0}^{t} g(s) \mathrm{d} s\right)^{q} w(t) \mathrm{d} t\right]^{\frac{1}{q}} \leq C\left(\int_{0}^{\infty} f^{p_{1}} v_{1}\right)^{\frac{1}{p_{1}}}\left(\int_{0}^{\infty} g^{p_{2}} v_{2}\right)^{\frac{1}{p_{2}}}
$$

for all $f, g \in \mathscr{M}_{+}$. The covered range of exponents in there was $1<$ $p, q<\infty$. For some related results see also the references in $[\mathbf{1}]$.

The paper [1] motivated the work presented here. Indeed, here we consider a restricted version of (4) which may be called the bilinear Hardy inequality for nonincreasing functions and written in the form

$$
\left[\int_{0}^{\infty}\left(\int_{0}^{t} f^{*}(s) \mathrm{d} s \int_{0}^{t} g^{*}(s) \mathrm{d} s\right)^{q} \frac{w(t)}{t^{2 q}} \mathrm{~d} t\right]^{\frac{1}{q}} \leq C\left(\int_{0}^{\infty}\left(f^{*}\right)^{p_{1}} v_{1}\right)^{\frac{1}{p_{1}}}\left(\int_{0}^{\infty}\left(g^{*}\right)^{p_{2}} v_{2}\right)^{\frac{1}{p_{2}}} .
$$

Notice that $C_{(1)}$ is the least constant $C$ for which the above inequality holds for all $f, g \in \mathscr{M}$.

The proofs in $[\mathbf{1}]$ are based on the standard technique of discretization. Here, however, we choose a different approach. The idea is as follows. In the first step, let $g$ in (1) be fixed. Treating $C_{(1)}$ as the optimal constant in the embedding $\Lambda^{p_{1}}\left(v_{1}\right) \hookrightarrow \Gamma^{q}\left(\left(g^{* *}\right)^{q} w\right)$, one gets

$$
C_{(1)}=\sup _{g \in \mathscr{M}} \frac{\|\operatorname{Id}\|_{\Lambda^{p_{1}}\left(v_{1}\right) \rightarrow \Gamma^{q}\left(\left(g^{* *}\right)^{q} w\right)}}{\|g\|_{\Lambda^{p_{2}}\left(v_{2}\right)}} .
$$

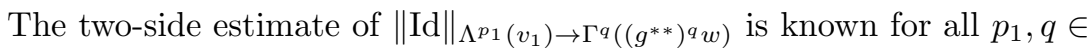
$(0, \infty]$ and it is equivalent to $\|g\|_{X}$, a certain rearrangement-invariant (quasi-)norm of $g$. Hence, in the next step, if we can find the optimal constant $\|\operatorname{Id}\|_{\Lambda^{p_{2}\left(v_{2}\right) \rightarrow X}}$, the whole problem is solved.

It will be shown that $\|\cdot\|_{X}$ can be expressed as a sum of (quasi-)norms in the r.i. spaces $J^{\alpha, \beta}(\varphi, \psi)$ and $K^{\alpha, \beta}(\varphi, \psi)$ (see Section 2 for the definitions). In Section 3 we find characterizations of the embeddings $\Lambda^{\gamma}(\omega) \hookrightarrow J^{\alpha, \beta}(\varphi, \psi)$ and $\Lambda^{\gamma}(\omega) \hookrightarrow K^{\alpha, \beta}(\varphi, \psi)$ for $0<\alpha \leq \beta<\infty$ 
and $0<\gamma<\infty$. In other words, we characterize the weights and exponents such that the inequalities

$$
\begin{aligned}
& \left(\int_{0}^{\infty}\left(\int_{0}^{x}\left(g^{* *}(t)\right)^{\alpha} \varphi(t) \mathrm{d} t\right)^{\frac{\beta}{\alpha}} \psi(x) \mathrm{d} x\right)^{\frac{1}{\beta}} \leq C\left(\int_{0}^{\infty}\left(g^{*}(x)\right)^{\gamma} \omega(x) \mathrm{d} x\right)^{\frac{1}{\gamma}}, \\
& \left(\int_{0}^{\infty}\left(\int_{x}^{\infty}\left(g^{* *}(t)\right)^{\alpha} \varphi(t) \mathrm{d} t\right)^{\frac{\beta}{\alpha}} \psi(x) \mathrm{d} x\right)^{\frac{1}{\beta}} \leq C\left(\int_{0}^{\infty}\left(g^{*}(x)\right)^{\gamma} \omega(x) \mathrm{d} x\right)^{\frac{1}{\gamma}}
\end{aligned}
$$

hold for all functions $g \in \mathscr{M}$. These results will be then used to find the desired estimates of the optimal constant $C_{(1)}$ in the bilinear Hardy inequality (this is the matter of Section 4). However, the description of the relation of the $K$-spaces to the other types of r.i. spaces, as well as the above weighted inequalities, are of independent interest.

\section{Auxiliary results}

Here we present various, usually known propositions which will be useful further on. First we may recall the following simple but useful principle. Let $a, b \in[-\infty, \infty]$ and let $f, g$ be nonnegative continuous functions on $(a, b), f$ nondecreasing, and $g$ nonincreasing. Then the derivatives $f^{\prime}(x), g^{\prime}(x)$ exist at a.e. $x \in(a, b)$. Denote $f(a+):=\lim _{x \rightarrow a+} f(x)$, $f(b-):=\lim _{x \rightarrow b-} f(x)$, similarly for $g$. Integration by parts then gives

$$
\int_{a}^{b} f^{\prime}(x) g(x) \mathrm{d} x+f(a+) g(a+)=f(b-) g(b-)-\int_{a}^{b} f(x) g^{\prime}(x) \mathrm{d} x,
$$

with the convention " $0 . \infty:=0$ " taking effect if needed. Thus, if we, for instance, consider $a:=0, b:=\infty, f:=W^{\alpha}, g:=V^{-\beta}$, and $\alpha, \beta \in$ $(0, \infty)$, we get

$$
\begin{aligned}
\int_{0}^{\infty} W^{\alpha-1}(x) w(x) V^{-\beta}(x) \mathrm{d} x \simeq W^{\alpha}(\infty) & V^{-\beta}(\infty) \\
& +\int_{0}^{\infty} W^{\alpha}(x) V^{-\beta-1}(x) v(x) \mathrm{d} x .
\end{aligned}
$$

Analogous situations arise if we take $f(x):=\left(\int_{x}^{\infty} w\right)^{\alpha}$, etc. However, if $\alpha<1$, there might appear a certain problem related to the integrability of the involved functions (cf. [28, p. 93]). Observe that if we take $\alpha \in$ $(0,1)$ in $(5)$ and a function $w \in \mathscr{M}_{+}$which is not integrable near the origin, then the equivalence in (5) fails, as the left-hand side is equal to zero while the right-hand side is infinite. Since we originally assumed 
that $w$ was a weight, which is by definition integrable near the origin, this problem, in fact, could not arise in (5). It may nevertheless do so in other situations when the involved function is not a weight in this sense and which thus require slightly more attention. We return to this issue in Proposition 2.3 below.

Anyway, combining or splitting weighted conditions using integration by parts in the described way is a common trick (see e.g. [30, Lemma, p. 176]). If there is no potential danger as described above (e.g. if the relevant exponents are greater than 1 ), we will use the technique throughout the text without detailed comments, and we will refer to it simply as to integration by parts.

Another well-known principle, to which we refer as to the $L^{p}$-duality, is expressed as follows. If $f \in \mathscr{M}_{+}, p \in(1, \infty)$ and $v$ is a weight, then

$$
\left(\int_{0}^{\infty} f^{p}(x) v(x) \mathrm{d} x\right)^{\frac{1}{p}}=\sup _{g \in \mathscr{M}_{+}} \frac{\int_{0}^{\infty} f(x) g(x) \mathrm{d} x}{\left(\int_{0}^{\infty} g^{p^{\prime}}(x) v^{1-p^{\prime}}(x) \mathrm{d} x\right)^{\frac{1}{p^{\prime}}}} .
$$

We continue with other preliminary results.

Proposition 2.1. Let $f, g \in \mathscr{M}_{+}$and $0<\lambda<\infty$. Then the identity

$$
\frac{\mathrm{d}}{\mathrm{d} x}\left[\int_{0}^{x}\left(\int_{s}^{x} f(t) \mathrm{d} t\right)^{\lambda} g(s) \mathrm{d} s\right]=\lambda f(x) \int_{0}^{x}\left(\int_{s}^{x} f(t) \mathrm{d} t\right)^{\lambda-1} g(s) \mathrm{d} s
$$

holds for a.e. $x>0$ for which the integral on the left-hand side is finite. Analogously, the identity

$$
\frac{\mathrm{d}}{\mathrm{d} x}\left[\int_{x}^{\infty}\left(\int_{x}^{s} f(t) \mathrm{d} t\right)^{\lambda} g(s) \mathrm{d} s\right]=-\lambda f(x) \int_{x}^{\infty}\left(\int_{x}^{s} f(t) \mathrm{d} t\right)^{\lambda-1} g(s) \mathrm{d} s
$$

holds for a.e. $x>0$ for which the integral on the left-hand side is finite.

Proof: Let us prove the first statement, the second one is analogous. Let

$$
x_{0}:=\sup \left\{x \in[0, \infty] ; \int_{0}^{x}\left(\int_{s}^{x} f(t) \mathrm{d} t\right)^{\lambda} g(s) \mathrm{d} s<\infty\right\} .
$$

Then, for any $x \in\left[0, x_{0}\right)$, Fubini theorem yields

$$
\begin{aligned}
\int_{0}^{x}\left(\int_{s}^{x} f(t) \mathrm{d} t\right)^{\lambda} g(s) \mathrm{d} s & =\int_{0}^{x}\left[\int_{s}^{x} \lambda\left(\int_{s}^{y} f(t) \mathrm{d} t\right)^{\lambda-1} f(y) \mathrm{d} y\right] g(s) \mathrm{d} s \\
& =\lambda \int_{0}^{x} f(y) \int_{0}^{y}\left(\int_{s}^{y} f(t) \mathrm{d} t\right)^{\lambda-1} g(s) \mathrm{d} s \mathrm{~d} y .
\end{aligned}
$$


The expression on the second line is nondecreasing and continuous in $x$, therefore its derivative with respect to $x$ exists and is equal to $\lambda f(x) \int_{0}^{x}\left(\int_{s}^{x} f(t) \mathrm{d} t\right)^{\lambda-1} g(s) \mathrm{d} s$ at a.e. point $x \in\left(0, x_{0}\right)$.

Proposition 2.2. Let $0<p \leq q<\infty$ and let $v, w$ be weights. Then it holds

$$
\sup _{\substack{\varphi \in \mathscr{M}_{+}+\text {is ing } \\ \varphi \text { indecreasing }}} \frac{\left(\int_{0}^{\infty} \varphi^{q}(x) w(x) \mathrm{d} x\right)^{\frac{1}{q}}}{\left(\int_{0}^{\infty} \varphi^{p}(x) v(x) \mathrm{d} x\right)^{\frac{1}{p}}} \simeq \sup _{x>0}\left(\int_{x}^{\infty} w\right)^{\frac{1}{q}}\left(\int_{x}^{\infty} v\right)^{-\frac{1}{p}} .
$$

Proof: This statement is analogous to a similar statement for nonincreasing functions (see [7, Theorem 3.1]). From there it can be also obtained directly by the change of variables $x \mapsto \frac{1}{x}$ in the integrals.

Proposition 2.3. Let $1<p<\infty$ and $0<q<p<\infty$. Let $v, w$ be weights. Then

$$
C_{(6)}:=\sup _{f \in \mathscr{M}} \frac{\left(\int_{0}^{\infty}\left(f^{* *}(t)\right)^{q} w(t) \mathrm{d} t\right)^{\frac{1}{q}}}{\left(\int_{0}^{\infty}\left(f^{*}(t)\right)^{p} v(t) \mathrm{d} t\right)^{\frac{1}{p}}} \simeq A_{(7)}+A_{(8)},
$$

where

$$
\begin{aligned}
A_{(7)}:=\left[\int_{0}^{\infty}\left(\frac{W(t)}{V(t)}\right)^{\frac{q}{p-q}} w(t) \mathrm{d} t\right]^{\frac{p-q}{p q}} \\
\\
\simeq\left[\int_{0}^{\infty}\left(\frac{W(t)}{V(t)}\right)^{\frac{p}{p-q}} v(t) \mathrm{d} t\right]^{\frac{p-q}{p q}}+W^{\frac{1}{q}}(\infty) V^{-\frac{1}{p}}(\infty)
\end{aligned}
$$

and

(8) $\quad A_{(8)}:=\left[\int_{0}^{\infty}\left(\int_{t}^{\infty} \frac{w(s)}{s^{q}} \mathrm{~d} s\right)^{\frac{q}{p-q}}\left(\int_{0}^{t} \frac{v(s) s^{p^{\prime}}}{V^{p^{\prime}}(s)} \mathrm{d} s\right)^{\frac{(p-1) q}{p-q}} \frac{w(t)}{t^{q}} \mathrm{~d} t\right]^{\frac{p-q}{p q}}$.

In particular, if $C_{(6)}<\infty$, then the function $s \mapsto v(s) s^{p^{\prime}} V^{-p^{\prime}}(s)$ is integrable near the origin.

Furthermore, if $q>1$, or if $q<1$ and the function $s \mapsto v(s) s^{p^{\prime}} V^{-p^{\prime}}(s)$ is integrable near the origin, then $A_{(8)} \simeq A_{(9)}$, where

$$
A_{(9)}:=\left[\int_{0}^{\infty}\left(\int_{t}^{\infty} \frac{w(s)}{s^{q}} \mathrm{~d} s\right)^{\frac{p}{p-q}}\left(\int_{0}^{t} \frac{v(s) s^{p^{\prime}}}{V^{p^{\prime}}(s)} \mathrm{d} s\right)^{\frac{(q-1) p}{p-q}} \frac{v(t) t^{p^{\prime}}}{V^{p^{\prime}}(t)} \mathrm{d} t\right]^{\frac{p-q}{p q}} .
$$


Proof: This assertion is stated in [7, Theorem 4.1(iii)] under the additional condition that $q \neq 1$. However, it is true even for $q=1$, which may be checked using [11, Theorem 3.1(iv)] and [14, Theorem 3.1].

Let us say more on the equivalence $A_{(8)} \simeq A_{(9)}$. If $q>1$ and the function $u$, defined by $u(s):=v(s) s^{p^{\prime}} V^{-p^{\prime}}(s)$ for $s>0$, is not integrable near the origin (a simple example of such function $u$ was given in $[\mathbf{2 8}$, p. 93]), then both $A_{(8)}$ and $A_{(9)}$ are infinite. However, if $q<1$ and $u$ is not integrable near the origin, then $A_{(8)}=\infty$ but $A_{(9)}=0$, since the exponent $\frac{(q-1) p}{p-q}$ is negative.

Proposition 2.3 will be later used e.g. in the proofs of Lemmas 3.2 and 3.3 and Theorem 4.3. In the calculations within the proofs, we will need to use conditions in the form of $A_{(9)}$. The reason is that the function involving $w$ appears only once in there and the resulting expression may be understood as the (quasi-)norm in a certain space. Nevertheless, for the final conditions which we state in the lemmas or theorems, we prefer the "safe" form in the style of $A_{(8)}$, i.e. avoiding the potentially negative exponents. In this way, the finiteness of the condition automatically implies the integrability of the "problematic" function near the origin.

The proposition below is a modification of [29, Proposition 2.7].

Proposition 2.4. Let $\|\cdot\|_{X}$ be a functional acting on $\mathscr{M}_{+}$such that for all $\lambda>0$ and all $g, h \in \mathscr{M}_{+}$such that $g \leq h$ a.e. it holds $\|g\|_{X} \leq\|h\|_{X}$ and $\|\lambda g\|_{X} \leq \lambda\|g\|_{X}$. Let $v$ be a weight. Then

$$
\sup _{f \in \mathscr{M}} \frac{\left\|f^{*}\right\|_{X}}{\|f\|_{\Lambda^{\infty}(v)}}=\left\|\left(\operatorname{esssup}_{y \in(0, \bullet)} v(y)\right)^{-1}\right\|_{X} .
$$

Proof: Let $f^{*} \in \mathscr{M}$. Then, by the properties of $\|\cdot\|_{X}$, one has

$$
\begin{aligned}
& \left\|f^{*}\right\|_{X} \leq \operatorname{esssup}_{x>0} f^{*}(x) \underset{y \in(0, x)}{\operatorname{essup} v} v(y)\left\|\left(\operatorname{esssup}_{y \in(0, \bullet)}^{\operatorname{ess} v(y)}\right)^{-1}\right\|_{X} \\
& =\underset{y>0}{\operatorname{ess} \sup } v(y) \underset{x \in(y, \infty)}{\operatorname{esssup}} f^{*}(x)\left\|\left(\operatorname{essup}_{y \in(0, \bullet)} v(y)\right)^{-1}\right\|_{X} \\
& =\|f\|_{\Lambda^{\infty}(v)}\left\|\left(\operatorname{esssup}_{y \in(0, \bullet)} v(y)\right)^{-1}\right\|_{X} .
\end{aligned}
$$

Taking the supremum over $f \in \mathscr{M}$, we get the inequality " $\leq$ " in (10).

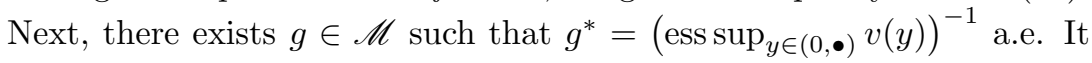


is easy to observe that

$$
\|g\|_{\Lambda^{\infty}(v)}=\operatorname{esssup}_{x>0} v(x)\left(\operatorname{esssup}_{y \in(0, x)} v(y)\right)^{-1}=1 .
$$

Hence, it holds $\frac{\left\|g^{*}\right\|_{X}}{\|g\|_{\Lambda}^{\infty}(v)}=\left\|g^{*}\right\|_{X}=\left\|\left(\operatorname{ess} \sup _{y \in(0, \bullet)} v(y)\right)^{-1}\right\|_{X}$ and thus the " $\geq$ " inequality in (10) is satisfied.

\section{Embeddings}

In this section we characterize certain embeddings $\Lambda \hookrightarrow J$ and $\Lambda \hookrightarrow K$. These results will later form a crucial step in the proof of the bilinear Hardy inequality.

At first, observe that the embedding $\Lambda^{\gamma}(\omega) \rightarrow K^{\alpha, \infty}(\varphi, \psi)$ is characterized easily by rephrasing the problem as an embedding $\Lambda \hookrightarrow \Gamma$.

Proposition 3.1. Let $\varphi, \psi, \omega$ be weights and $0<\alpha, \beta, \gamma \leq \infty$. Then

$$
\|\operatorname{Id}\|_{\Lambda^{\gamma}(\omega) \rightarrow K^{\alpha, \infty}(\varphi, \psi)}=\underset{x>0}{\operatorname{ess} \sup } \psi(x)\|\operatorname{Id}\|_{\Lambda^{\gamma}(\omega) \rightarrow \Gamma^{\alpha}\left(\varphi \chi_{[x, \infty)}\right)} .
$$

Proof: We have

$$
\begin{aligned}
\sup _{g \in \mathscr{M}} \operatorname{ess} \sup _{x>0} \frac{\left(\int_{x}^{\infty}\left(g^{* *}\right)^{\alpha} \varphi\right)^{\frac{1}{\alpha}} \psi(x)}{\left(\int_{0}^{\infty}\left(g^{*}\right)^{\gamma} \omega\right)^{\frac{1}{\gamma}}} & =\operatorname{esssup}_{x>0} \psi(x) \sup _{g \in \mathscr{M}} \frac{\left(\int_{x}^{\infty}\left(g^{* *}\right)^{\alpha} \varphi\right)^{\frac{1}{\alpha}}}{\left(\int_{0}^{\infty}\left(g^{*}\right)^{\gamma} \omega\right)^{\frac{1}{\gamma}}} \\
& =\operatorname{essup}_{x>0} \psi(x)\|\operatorname{Id}\|_{\Lambda^{\gamma}(\omega) \rightarrow \Gamma^{\alpha}\left(\varphi \chi_{[x, \infty)}\right)} .
\end{aligned}
$$

The embeddings $\Lambda \hookrightarrow \Gamma$ have been fully characterized (see $[\mathbf{7}, \mathbf{6}]$ ). Similarly it can be dealt with the embedding $\Lambda^{\gamma}(\omega) \rightarrow J^{\alpha, \infty}(\varphi, \psi)$, where the problem reduces to a characterization the boundedness of the dual Hardy operator on the cone of nonincreasing functions. Results regarding the latter problem are also at our disposal, se e.g. [17].

Recall that if $\varphi, \psi, \omega$ are weights, then $\Phi(t):=\int_{0}^{t} \varphi, \Psi(t):=\int_{0}^{t} \psi$, $\Omega(t):=\int_{0}^{t} \omega$ for $t>0$. In the couple of lemmas below there will appear a function $\sigma$, defined by

$$
\sigma(x):=\sup _{t \in(0, x)}\left(t \Omega^{-\frac{1}{\gamma}}(t)\right)^{\frac{\gamma \alpha}{\gamma-\alpha}}, \quad x>0,
$$

where $\omega$ is a weight and $\alpha, \gamma \in(0, \infty)$ are exponents specified later. The function $\sigma$ is continuous and nondecreasing on $(0, \infty)$, hence its derivative $\sigma^{\prime}$ exists at almost every point $x>0$ and, furthermore, for all $x>0$ it holds $\sigma(x)=\int_{0}^{x} \sigma^{\prime}(t) \mathrm{d} t+\sigma(0+)$, where $\sigma(0+):=$ 
$\lim \sup _{t \rightarrow 0+}\left(t \Omega^{-\frac{1}{\gamma}}(t)\right)^{\frac{\gamma \alpha}{\gamma-\alpha}}$. This notation and properties of $\sigma$ are used in the lemmas without further comment.

The lemma below brings a characterization of the embedding $\Lambda^{\gamma}(\omega) \hookrightarrow$ $J^{\alpha, \beta}(\varphi, \psi)$ for $0<\alpha \leq \beta<\infty$ and $\alpha<\gamma<\infty$.

Lemma 3.2. Let $\varphi, \psi, \omega$ be weights. Denote

$$
C_{(12)}:=\sup _{g \in \mathscr{M}} \frac{\left(\int_{0}^{\infty}\left(\int_{0}^{x}\left(g^{* *}\right)^{\alpha} \varphi\right)^{\frac{\beta}{\alpha}} \psi(x) \mathrm{d} x\right)^{\frac{1}{\beta}}}{\left(\int_{0}^{\infty}\left(g^{*}\right)^{\gamma} \omega\right)^{\frac{1}{\gamma}}} .
$$

(i) Let $0<\alpha<\gamma \leq \beta<\infty$ and $1<\gamma$. Then $C_{(12)} \simeq A_{(13)}+A_{(14)}$, where

$$
A_{(13)}:=\sup _{x>0} \Omega^{-\frac{1}{\gamma}}(x)\left(\int_{0}^{x} \Phi^{\frac{\beta}{\alpha}} \psi\right)^{\frac{1}{\beta}}+\sup _{x>0}\left(\int_{0}^{x} \Phi^{\frac{\gamma}{\gamma-\alpha}} \Omega^{\frac{\gamma}{\alpha-\gamma}} \omega\right)^{\frac{\gamma-\alpha}{\gamma \alpha}}\left(\int_{x}^{\infty} \psi\right)^{\frac{1}{\beta}}
$$

and

$$
\begin{aligned}
A_{(14)}:= & \sup _{x>0}\left[\int_{0}^{x}\left(\int_{s}^{x} \frac{\varphi(t)}{t^{\alpha}} \mathrm{d} t\right)^{\frac{\alpha}{\gamma-\alpha}} \frac{\varphi(s)}{s^{\alpha}}\left(\int_{0}^{s} \frac{y^{\gamma^{\prime}} \omega(y)}{\Omega^{\gamma^{\prime}}(y)} \mathrm{d} y\right)^{\frac{\alpha(\gamma-1)}{\gamma-\alpha}} \mathrm{d} s\right]^{\frac{\gamma-\alpha}{\gamma \alpha}}\left(\int_{x}^{\infty} \psi\right)^{\frac{1}{\beta}} \\
& +\sup _{x>0}\left[\int_{x}^{\infty}\left(\int_{x}^{s} \frac{\varphi(t)}{t^{\alpha}} \mathrm{d} t\right)^{\frac{\beta}{\alpha}} \psi(s) \mathrm{d} s\right]^{\frac{1}{\beta}}\left(\int_{0}^{x} \frac{s^{\gamma^{\prime}} \omega(s)}{\Omega^{\gamma^{\prime}}(s)} \mathrm{d} s\right)^{\frac{\gamma-1}{\gamma}} .
\end{aligned}
$$

(ii) Let $0<\alpha<\beta<\gamma<\infty$ and $1<\gamma$. Then $C_{(12)} \simeq A_{(15)}+A_{(16)}$, where

$$
\begin{aligned}
A_{(15)}:= & {\left[\int_{0}^{\infty} \Omega^{\frac{\beta}{\beta-\gamma}}(x)\left(\int_{0}^{x} \Phi^{\frac{\beta}{\alpha}} \psi\right)^{\frac{\beta}{\gamma-\beta}} \Phi^{\frac{\beta}{\alpha}}(x) \psi(x) \mathrm{d} x\right]^{\frac{\gamma-\beta}{\gamma \beta}} } \\
& +\left[\int_{0}^{\infty}\left(\int_{0}^{x} \Phi^{\frac{\gamma}{\gamma-\alpha}} \Omega^{\frac{\gamma}{\alpha-\gamma}} \omega\right)^{\frac{\gamma(\beta-\alpha)}{\alpha(\gamma-\beta)}} \Phi^{\frac{\gamma}{\gamma-\alpha}}(x) \Omega^{\frac{\gamma}{\alpha-\gamma}}(x) \omega(x)\left(\int_{x}^{\infty} \psi\right)^{\frac{\gamma}{\gamma-\beta}} \mathrm{d} x\right]^{\frac{\gamma-\beta}{\gamma \beta}}
\end{aligned}
$$


and

$$
\text { (16) } \begin{aligned}
A_{(16):=} & {\left[\int _ { 0 } ^ { \infty } \left(\int_{0}^{x}\left(\int_{s}^{x} \frac{\varphi(t)}{t^{\alpha}} \mathrm{d} t\right)^{\frac{\alpha}{\gamma-\alpha}} \frac{\varphi(s)}{s^{\alpha}}\right.\right.} \\
& \left.\left.\times\left(\int_{0}^{s} \frac{y^{\gamma^{\prime}} \omega(y)}{\Omega^{\gamma^{\prime}}(y)} \mathrm{d} y\right)^{\frac{\alpha(\gamma-1)}{\gamma-\alpha}} \mathrm{d} s\right)^{\frac{\beta(\gamma-\alpha)}{\alpha(\gamma-\beta)}}\left(\int_{x}^{\infty} \psi\right)^{\frac{\beta}{\gamma-\beta}} \psi(x) \mathrm{d} x\right]^{\frac{\gamma-\beta}{\gamma \beta}} \\
+ & {\left[\int_{0}^{\infty}\left(\int_{x}^{\infty}\left(\int_{x}^{s} \frac{\varphi(t)}{t^{\alpha}} \mathrm{d} t\right)^{\frac{\beta}{\alpha}} \psi(s) \mathrm{d} s\right)^{\frac{\beta}{\gamma-\beta}} \frac{\varphi(x)}{x^{\alpha}}\right.} \\
& \left.\times \int_{x}^{\infty}\left(\int_{x}^{y} \frac{\varphi(t)}{t^{\alpha}} \mathrm{d} t\right)^{\frac{\beta-\alpha}{\alpha}} \psi(y) \mathrm{d} y\left(\int_{0}^{x} \frac{s^{\gamma^{\prime}} \omega(s)}{\Omega^{\gamma^{\prime}}(s)} \mathrm{d} s\right)^{\frac{\beta(\gamma-1)}{\gamma-\beta}} \mathrm{d} x\right]^{\frac{\gamma-\beta}{\gamma \beta}} .
\end{aligned}
$$

(iii) Let $0<\alpha<\gamma \leq \beta<\infty$ and $\gamma \leq 1$. Let $\sigma$ be given by (11). Then $C_{(12)} \simeq A_{(13)}+A_{(17)}$, where

$$
\begin{aligned}
A_{(17)}:= & \sup _{x>0}\left[\int_{0}^{x}\left(\int_{s}^{x} \frac{\varphi(t)}{t^{\alpha}} \mathrm{d} t\right)^{\frac{\alpha}{\gamma-\alpha}} \frac{\varphi(s)}{s^{\alpha}} \sigma(s) \mathrm{d} s\right]^{\frac{\gamma-\alpha}{\gamma \alpha}}\left(\int_{x}^{\infty} \psi\right)^{\frac{1}{\beta}} \\
& +\sup _{x>0} \sigma^{\frac{\gamma-\alpha}{\gamma \alpha}}(x)\left[\int_{x}^{\infty}\left(\int_{x}^{s} \frac{\varphi(t)}{t^{\alpha}} \mathrm{d} t\right)^{\frac{\beta}{\alpha}} \psi(s) \mathrm{d} s\right]^{\frac{1}{\beta}} .
\end{aligned}
$$

(iv) Let $0<\alpha<\beta<\gamma \leq 1$. Let $\sigma$ be given by (11). Then $C_{(12)} \simeq$ $A_{(15)}+A_{(18)}+A_{(19)}$, where

$$
\begin{aligned}
A_{(18)}:=\left[\int_{0}^{\infty}\left(\int_{0}^{x}\left(\int_{s}^{x} \frac{\varphi(t)}{t^{\alpha}} \mathrm{d} t\right)^{\frac{\alpha}{\gamma-\alpha}} \frac{\varphi(s)}{s^{\alpha}} \sigma(s) \mathrm{d} s\right)^{\frac{\beta(\gamma-\alpha)}{\alpha(\gamma-\beta)}}\right. \\
\left.\quad \times\left(\int_{x}^{\infty} \psi\right)^{\frac{\beta}{\gamma-\beta}} \psi(x) \mathrm{d} x\right]^{\frac{\gamma-\beta}{\gamma \beta}}
\end{aligned}
$$

and

$$
\begin{aligned}
A_{(19)}:= & {\left[\int_{0}^{\infty}\left(\int_{x}^{\infty}\left(\int_{x}^{s} \frac{\varphi(t)}{t^{\alpha}} \mathrm{d} t\right)^{\frac{\beta}{\alpha}} \psi(s) \mathrm{d} s\right)^{\frac{\beta}{\gamma-\beta}}\right.} \\
& \left.\times \int_{x}^{\infty}\left(\int_{x}^{s} \frac{\varphi(t)}{t^{\alpha}} \mathrm{d} t\right)^{\frac{\beta-\alpha}{\alpha}} \psi(s) \mathrm{d} s \frac{\varphi(x)}{x^{\alpha}} \sigma^{\frac{\beta(\gamma-\alpha)}{\alpha(\gamma-\beta)}}(x) \mathrm{d} x\right]^{\frac{\gamma-\beta}{\gamma \beta}} .
\end{aligned}
$$


Proof: We have

$$
\begin{aligned}
C_{(12)}= & \sup _{g \in \mathscr{M}} \sup _{h \in \mathscr{M}_{+}} \frac{1}{\left(\int_{0}^{\infty} h^{\frac{\beta}{\beta-\alpha}} \psi^{\frac{\alpha}{\alpha-\beta}}\right)^{\frac{\beta-\alpha}{\beta \alpha}}} \\
& \times \frac{\left(\int_{0}^{\infty} h(x) \int_{0}^{x}\left(g^{* *}(t)\right)^{\alpha} \varphi(t) \mathrm{d} t \mathrm{~d} x\right)^{\frac{1}{\alpha}}}{\left(\int_{0}^{\infty}\left(g^{*}\right)^{\gamma} \omega\right)^{\frac{1}{\gamma}}} \\
= & \sup _{h \in \mathscr{M}_{+}} \frac{1}{\left(\int_{0}^{\infty} h^{\frac{\beta}{\beta-\alpha}} \psi^{\frac{\alpha}{\alpha-\beta}}\right)^{\frac{\beta-\alpha}{\beta \alpha}}} \\
& \times \sup _{g \in \mathscr{M}} \frac{\left(\int_{0}^{\infty}\left(g^{* *}(t)\right)^{\alpha} \varphi(t) \int_{t}^{\infty} h(x) \mathrm{d} x \mathrm{~d} t\right)^{\frac{1}{\alpha}}}{\left(\int_{0}^{\infty}\left(g^{*}\right)^{\gamma} \omega\right)^{\frac{1}{\gamma}}} \\
= & : B_{0} .
\end{aligned}
$$

In step (20) we used duality of $L^{p}$-spaces and (21) follows by Fubini theorem and changing the order of the suprema.

To make the notation shorter, define the function $u$ by

$$
u(s):=\frac{s^{\gamma^{\prime}} \omega(s)}{\Omega^{\gamma^{\prime}}(s)}, \quad s>0
$$

Now suppose that $\gamma>1$. Assume that $u$ is integrable near the origin. Then by Proposition 2.3 it holds

$$
\begin{aligned}
& B_{0} \simeq \sup _{h \in \mathscr{M}_{+}} \frac{\left(\int_{0}^{\infty}\left(\int_{0}^{s} \varphi(t) \int_{t}^{\infty} h(x) \mathrm{d} x \mathrm{~d} t\right)^{\frac{\gamma}{\gamma-\alpha}} \Omega^{\frac{\gamma}{\alpha-\gamma}}(s) \omega(s) \mathrm{d} s\right)^{\frac{\gamma-\alpha}{\gamma \alpha}}}{\left(\int_{0}^{\infty} h^{\frac{\beta}{\beta-\alpha}} \psi^{\frac{\alpha}{\alpha-\beta}}\right)^{\frac{\beta-\alpha}{\beta \alpha}}} \\
& \quad+\sup _{h \in \mathscr{M}_{+}} \frac{\left(\int_{0}^{\infty} \varphi(t) \int_{t}^{\infty} h(x) \mathrm{d} x \mathrm{~d} t\right)^{\frac{1}{\alpha}}}{\left(\int_{0}^{\infty} h^{\frac{\beta}{\beta-\alpha}} \psi^{\frac{\alpha}{\alpha-\beta}}\right)^{\frac{\beta-\alpha}{\beta \alpha}} \Omega^{\frac{1}{\gamma}}(\infty)} \\
& \quad+\sup _{h \in \mathscr{M}_{+}} \frac{\left(\int_{0}^{\infty}\left(\int_{s}^{\infty} \frac{\varphi(t)}{t^{\alpha}} \int_{t}^{\infty} h(x) \mathrm{d} x \mathrm{~d} t\right)^{\frac{\gamma}{\gamma-\alpha}}\left(\int_{0}^{s} u(y) \mathrm{d} y\right)^{\frac{\gamma(\alpha-1)}{\gamma-\alpha}} u(s) \mathrm{d} s\right)^{\frac{\gamma-\alpha}{\gamma \alpha}}}{\left(\int_{0}^{\infty} h^{\frac{\beta}{\beta-\alpha}} \psi^{\frac{\alpha}{\alpha-\beta}}\right)^{\frac{\beta-\alpha}{\beta \alpha}}} \\
&=: B_{1}+B_{2}+B_{3} .
\end{aligned}
$$


Consider now the case (i). It holds

$$
\begin{aligned}
B_{1} \simeq \sup _{h \in \mathscr{M}_{+}} \frac{\left(\int_{0}^{\infty}\left(\int_{0}^{s} h(x) \mathrm{d} x\right)^{\frac{\gamma}{\gamma-\alpha}} \Omega^{\frac{\gamma}{\alpha-\gamma}}(s) \omega(s) \mathrm{d} s\right)^{\frac{\gamma-\alpha}{\gamma \alpha}}}{\left(\int_{0}^{\infty} h^{\frac{\beta}{\beta-\alpha}} \Phi^{\frac{\beta}{\alpha-\beta}} \psi^{\frac{\alpha}{\alpha-\beta}}\right)^{\frac{\beta-\alpha}{\beta \alpha}}} \\
\quad+\sup _{h \in \mathscr{M}_{+}} \frac{\left(\int_{0}^{\infty}\left(\int_{s}^{\infty} h(x) \mathrm{d} x\right)^{\frac{\gamma}{\gamma-\alpha}} \Phi^{\frac{\gamma}{\gamma-\alpha}}(s) \Omega^{\frac{\gamma}{\alpha-\gamma}}(s) \omega(s) \mathrm{d} s\right)^{\frac{\gamma-\alpha}{\gamma \alpha}}}{\left(\int_{0}^{\infty} h^{\frac{\beta}{\beta-\alpha}} \psi^{\frac{\alpha}{\alpha-\beta}}\right)^{\frac{\beta-\alpha}{\beta \alpha}}} \\
\simeq \sup _{x>0}\left(\int_{x}^{\infty} \Omega^{\frac{\gamma}{\alpha-\gamma} \omega}\right)^{\frac{\gamma-\alpha}{\gamma \alpha}}\left(\int_{0}^{x} \Phi^{\frac{\beta}{\alpha}} \psi\right)^{\frac{1}{\beta}} \\
\quad+\sup _{x>0}\left(\int_{0}^{x} \Phi^{\frac{\gamma}{\gamma-\alpha}} \Omega^{\frac{\gamma}{\alpha-\gamma} \omega}\right)^{\frac{\gamma-\alpha}{\gamma \alpha}}\left(\int_{x}^{\infty} \psi\right)^{\frac{1}{\beta}},
\end{aligned}
$$

where (23) follows by Fubini theorem and (24) by Hardy inequality (see $\left[21\right.$, p. 3-4]). Next, Fubini theorem and $L^{p}$-duality yield

$$
B_{2} \simeq\left(\int_{0}^{\infty} \Phi^{\frac{\beta}{\alpha}} \psi\right)^{\frac{1}{\beta}} \Omega^{-\frac{1}{\gamma}}(\infty)=\sup _{x>0}\left(\int_{0}^{x} \Phi^{\frac{\beta}{\alpha}} \psi\right)^{\frac{1}{\beta}}\left(\Omega^{\frac{\alpha}{\alpha-\gamma}}(\infty)\right)^{\frac{\gamma-\alpha}{\gamma \alpha}}
$$

Therefore, we have

$$
\begin{aligned}
B_{2}+B_{1} \simeq & \sup _{x>0}\left(\Omega^{\frac{\alpha}{\alpha-\gamma}}(\infty)+\int_{x}^{\infty} \Omega^{\frac{\gamma}{\alpha-\gamma}} \omega\right)^{\frac{\gamma-\alpha}{\gamma \alpha}}\left(\int_{0}^{x} \Phi^{\frac{\beta}{\alpha}} \psi\right)^{\frac{1}{\beta}} \\
& +\sup _{x>0}\left(\int_{0}^{x} \Phi^{\frac{\gamma}{\gamma-\alpha}} \Omega^{\frac{\gamma}{\alpha-\gamma} \omega}\right)^{\frac{\gamma-\alpha}{\gamma \alpha}}\left(\int_{x}^{\infty} \psi\right)^{\frac{1}{\beta}} \simeq A_{(13)} .
\end{aligned}
$$

Notice that this equivalence in fact does not involve the function $u$ at all, hence it holds for any $u \in \mathscr{M}_{+}$. The assumption on $u$ will be used only in the next part. By Fubini theorem, $B_{3}$ is equal to

$$
\sup _{h \in \mathscr{M}_{+}} \frac{\left[\int_{0}^{\infty}\left(\int_{s}^{\infty} h(x) \int_{s}^{x} \frac{\varphi(t)}{t^{\alpha}} \mathrm{d} t \mathrm{~d} x\right)^{\frac{\gamma}{\gamma-\alpha}}\left(\int_{0}^{s} u(y) \mathrm{d} y\right)^{\frac{\gamma(\alpha-1)}{\gamma-\alpha}} u(s) \mathrm{d} s\right]^{\frac{\gamma-\alpha}{\gamma \alpha}}}{\left(\int_{0}^{\infty} h^{\frac{\beta}{\beta-\alpha}} \psi^{\frac{\alpha}{\alpha-\beta}}\right)^{\frac{\beta-\alpha}{\beta \alpha}}} .
$$


This expression is, by the dual version of [24, Theorem 1.1], equivalent to

$$
\begin{array}{r}
\sup _{x>0}\left[\int_{0}^{x}\left(\int_{s}^{x} \frac{\varphi(t)}{t^{\alpha}} \mathrm{d} t\right)^{\frac{\gamma}{\gamma-\alpha}}\left(\int_{0}^{s} u(y) \mathrm{d} y\right)^{\frac{\gamma(\alpha-1)}{\gamma-\alpha}} u(s) \mathrm{d} s\right]^{\frac{\gamma-\alpha}{\gamma \alpha}}\left(\int_{x}^{\infty} \psi\right)^{\frac{1}{\beta}} \\
+\sup _{x>0}\left[\int_{x}^{\infty}\left(\int_{x}^{s} \frac{\varphi(t)}{t^{\alpha}} \mathrm{d} t\right)^{\frac{\beta}{\alpha}} \psi(s) \mathrm{d} s\right]^{\frac{1}{\beta}}\left(\int_{0}^{x} u(s) \mathrm{d} s\right)^{\frac{\gamma-1}{\gamma}},
\end{array}
$$

which is, in turn, equivalent to $A_{(14)}$ by Proposition 2.3 , since $u$ is integrable at the origin. Finally, observe that if $u$ is not integrable at the origin, then necessarily both $B_{0}=\infty$ (see the proof sketch of Proposition 2.3) and $A_{(14)}=\infty$. On the other hand, if $A_{(14)}<\infty$, then $u$ is integrable at the origin. Hence, $C_{(12)}=B_{0}<\infty$ holds if and only if $A_{(13)}+A_{(14)}<\infty$. Moreover, $C_{(12)} \simeq A_{(13)}+A_{(14)}$, all without any additional assumptions on the weight $u$.

In case (ii), using an appropriate version of Hardy inequality and

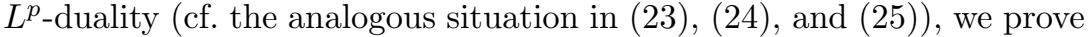
that $B_{1}+B_{2} \simeq A_{(15)}$. To estimate $B_{3}$, we use [24, Theorem 1.2]. Then we get

$$
\begin{array}{r}
B_{3} \simeq\left[\int_{0}^{\infty}\left(\int_{0}^{x}\left(\int_{s}^{x} \frac{\varphi(t)}{t^{\alpha}} \mathrm{d} t\right)^{\frac{\gamma}{\gamma-\alpha}}\left(\int_{0}^{s} \frac{y^{\gamma^{\prime}} \omega(y)}{\Omega^{\gamma^{\prime}}(y)} \mathrm{d} y\right)^{\frac{\gamma(\alpha-1)}{\gamma-\alpha}} \frac{s^{\gamma^{\prime}} \omega(s)}{\Omega^{\gamma^{\prime}}(s)} \mathrm{d} s\right)^{\frac{\beta(\gamma-\alpha)}{\alpha(\gamma-\beta)}}\right. \\
\left.\left.+\left[\int_{0}^{\infty}\left(\int_{x}^{\infty} \psi\right)^{\frac{\beta}{\gamma-\beta}} \psi(x) \mathrm{d} x\right]^{\frac{\gamma-\beta}{\gamma \beta}} \frac{\varphi(t)}{t^{\alpha}} \mathrm{d} t\right)^{\frac{\beta}{\alpha}} \psi(s) \mathrm{d} s\right)^{\frac{\gamma}{\gamma-\beta}} \\
\left.\times\left(\int_{0}^{x} \frac{s^{\gamma^{\prime}} \omega(s)}{\Omega^{\gamma^{\prime}}(s)} \mathrm{d} s\right)^{\frac{\gamma(\beta-1)}{\gamma-\beta}} \frac{x^{\gamma^{\prime}} \omega(x)}{\Omega^{\gamma^{\prime}}(x)} \mathrm{d} x\right]^{\frac{\gamma-\beta}{\gamma \beta}} .
\end{array}
$$

Using the assumption of integrability at the origin of $u$, one may show then by integration by parts that the above expression is equivalent to $A_{(16)}$. While handling the second term in the sum, one also needs to use Proposition 2.1. Finally, the additional assumption on $u$ is removed in the same way as in case (i). 
Now we assume $0<\gamma \leq 1$. From [6, Theorem 3.1] it follows that $B_{0}=B_{1}+B_{2}+B_{4}$, where

$B_{4}:=\sup _{h \in \mathscr{M}_{+}} \frac{\left[\int_{0}^{\infty} \sup _{0<t \leq s}\left(\frac{t}{V^{\frac{1}{\gamma}(t)}}\right)^{\frac{\gamma \alpha}{\gamma-\alpha}}\left(\int_{s}^{\infty} \frac{\varphi(t)}{t^{\alpha}} \int_{t}^{\infty} h(x) \mathrm{d} x \mathrm{~d} t\right)^{\frac{\alpha}{\gamma-\alpha}} \frac{\varphi(s)}{s^{\alpha}} \int_{s}^{\infty} h(x) \mathrm{d} x \mathrm{~d} s\right]^{\frac{\gamma-\alpha}{\gamma \alpha}}}{\left(\int_{0}^{\infty} h^{\frac{\beta}{\beta-\alpha}} \psi^{\frac{\alpha}{\alpha-\beta}}\right)^{\frac{\beta-\alpha}{\beta \alpha}}}$.

Furthermore,

$$
\begin{aligned}
& B_{4} \simeq \sup _{h \in \mathscr{M}_{+}} \frac{\left[\int_{0}^{\infty} \sigma^{\prime}(s)\left(\int_{s}^{\infty} \frac{\varphi(t)}{t^{\alpha}} \int_{t}^{\infty} h(x) \mathrm{d} x \mathrm{~d} t\right)^{\frac{\gamma}{\gamma-\alpha}} \mathrm{d} s\right]^{\frac{\gamma-\alpha}{\gamma \alpha}}}{\left(\int_{0}^{\infty} h^{\frac{\beta}{\beta-\alpha}} \psi^{\frac{\alpha}{\alpha-\beta}}\right)^{\frac{\beta-\alpha}{\beta \alpha}}} \\
&+\sup _{h \in \mathscr{M}_{+}} \frac{\sigma^{\frac{\gamma-\alpha}{\gamma \alpha}}(0+)\left(\int_{0}^{\infty} \frac{\varphi(s)}{s^{\alpha}} \int_{s}^{\infty} h(x) \mathrm{d} x \mathrm{~d} s\right)^{\frac{1}{\alpha}}}{\left(\int_{0}^{\infty} h^{\frac{\beta}{\beta-\alpha}} \psi^{\frac{\alpha}{\alpha-\beta}}\right)^{\frac{\beta-\alpha}{\beta \alpha}}} \\
&=\sup _{h \in \mathscr{M}_{+}} \frac{\left[\int_{0}^{\infty} \sigma^{\prime}(s)\left(\int_{s}^{\infty} h(x) \int_{s}^{x} \frac{\varphi(t)}{t^{\alpha}} \mathrm{d} t \mathrm{~d} x\right)^{\frac{\gamma}{\gamma-\alpha}} \mathrm{d} s\right]^{\frac{\gamma-\alpha}{\gamma \alpha}}}{\left(\int_{0}^{\infty} h^{\frac{\beta}{\beta-\alpha}} \psi^{\frac{\alpha}{\alpha-\beta}}\right)^{\frac{\beta-\alpha}{\beta \alpha}}} \\
& \quad+\sup _{h \in \mathscr{M}_{+}} \frac{\sigma^{\frac{\gamma-\alpha}{\gamma \alpha}}(0+)\left(\int_{0}^{\infty} h(x) \int_{0}^{x} \frac{\varphi(s)}{s^{\alpha}} \mathrm{d} s \mathrm{~d} x\right)^{\frac{1}{\alpha}}}{\left(\int_{0}^{\infty} h^{\frac{\beta}{\beta-\alpha}} \psi^{\frac{\alpha}{\alpha-\beta}}\right)^{\frac{\beta-\alpha}{\beta \alpha}}} \\
&=: B_{5}+B_{6} .
\end{aligned}
$$

For (26) one uses integration by parts and (27) follows by Fubini theorem. Next, by $L^{p}$-duality, we get

$$
B_{6}=\sigma^{\frac{\gamma-\alpha}{\gamma \alpha}}(0+)\left[\int_{0}^{\infty}\left(\int_{0}^{x} \frac{\varphi(s)}{s^{\alpha}} \mathrm{d} s\right)^{\frac{\beta}{\alpha}} \psi(x) \mathrm{d} x\right]^{\frac{1}{\beta}} .
$$


Consider now the case (iii). From the dual version of [24, Theorem 1.1] it follows

$$
\begin{aligned}
B_{5} \simeq \sup _{x>0}\left[\int_{0}^{x}\left(\int_{s}^{x} \frac{\varphi(t)}{t^{\alpha}} \mathrm{d} t\right)^{\frac{\gamma}{\gamma-\alpha}} \sigma^{\prime}(s) \mathrm{d} s\right]^{\frac{\gamma-\alpha}{\gamma \alpha}}\left(\int_{x}^{\infty} \psi\right)^{\frac{1}{\beta}} \\
+\sup _{x>0}\left(\int_{0}^{x} \sigma^{\prime}\right)^{\frac{\gamma-\alpha}{\gamma \alpha}}\left[\int_{x}^{\infty}\left(\int_{x}^{s} \frac{\varphi(t)}{t^{\alpha}} \mathrm{d} t\right)^{\frac{\beta}{\alpha}} \psi(s) \mathrm{d} s\right]^{\frac{1}{\beta}} .
\end{aligned}
$$

Using this characterization, the expression of $B_{6}$ from (28) and integrating by parts, one obtains $B_{5}+B_{6} \simeq A_{(17)}$. Earlier (when considering $\beta \geq \gamma>1$ ) we proved that $B_{1}+B_{2} \simeq A_{(13)}$. The same is true here, as the argument is correct even for $\beta \geq \gamma$ with $0<\gamma \leq 1$. Hence, it follows that $C_{(12)} \simeq B_{1}+B_{2}+B_{5}+B_{6} \simeq A_{(13)}+A_{(17)}$ and the proof of this part is complete.

We proceed with (iv). Estimating $B_{1}$ and $B_{2}$ is done in the same way as in (ii). It remains to show that $B_{5}+B_{6} \simeq A_{(18)}+A_{(19)}$. By the dual version of [24, Theorem 1.2], one has

$$
\begin{aligned}
B_{5} \simeq & {\left[\int_{0}^{\infty}\left(\int_{0}^{x}\left(\int_{s}^{x} \frac{\varphi(t)}{t^{\alpha}} \mathrm{d} t\right)^{\frac{\gamma}{\gamma-\alpha}} \sigma^{\prime}(s) \mathrm{d} s\right)^{\frac{\beta(\gamma-\alpha)}{\alpha(\gamma-\beta)}}\left(\int_{x}^{\infty} \psi\right)^{\frac{\beta}{\gamma-\beta}} \psi(x) \mathrm{d} x\right]^{\frac{\gamma-\beta}{\gamma \beta}} } \\
& +\left[\int_{0}^{\infty}\left(\int_{x}^{\infty}\left(\int_{x}^{s} \frac{\varphi(t)}{t^{\alpha}} \mathrm{d} t\right)^{\frac{\beta}{\alpha}} \psi(s) \mathrm{d} s\right)^{\frac{\gamma}{\gamma-\beta}}\left(\int_{0}^{x} \sigma^{\prime}\right)^{\frac{\gamma(\beta-\alpha)}{\alpha(\gamma-\beta)}} \sigma^{\prime}(x) \mathrm{d} x\right]^{\frac{\gamma-\beta}{\gamma \beta}} \\
= & : B_{7}+B_{8} .
\end{aligned}
$$

Now, integration by parts provides

(30) $A_{(18)} \simeq B_{7}+\sigma^{\frac{\gamma-\alpha}{\gamma \alpha}}(0+)\left[\int_{0}^{\infty}\left(\int_{0}^{x} \frac{\varphi(t)}{t^{\alpha}} \mathrm{d} t\right)^{\frac{\gamma \beta}{\alpha(\gamma-\beta)}}\left(\int_{x}^{\infty} \psi\right)^{\frac{\beta}{\gamma-\beta}} \psi(x) \mathrm{d} x\right]^{\frac{\gamma-\beta}{\gamma \beta}}$.

Next, it holds

$$
\sup _{x>0}\left[\int_{x}^{\infty}\left(\int_{t}^{\infty} \psi\right)^{\frac{\beta}{\gamma-\beta}} \psi(t) \mathrm{d} t\right]^{\frac{\alpha(\gamma-\beta)}{\gamma \beta}}\left(\int_{x}^{\infty} \psi\right)^{-\frac{\alpha}{\beta}} \simeq 1
$$


thus, by Proposition 2.2, we get

$$
\begin{aligned}
{\left[\int_{0}^{\infty}\left(\int_{0}^{x} \frac{\varphi(t)}{t^{\alpha}} \mathrm{d} t\right)^{\frac{\gamma \beta}{\alpha(\gamma-\beta)}}\left(\int_{x}^{\infty} \psi\right)^{\frac{\beta}{\gamma-\beta}} \psi(x) \mathrm{d} x\right]^{\frac{\alpha(\gamma-\beta)}{\gamma \beta}} } \\
\lesssim\left[\int_{0}^{\infty}\left(\int_{0}^{x} \frac{\varphi(s)}{s^{\alpha}} \mathrm{d} s\right)^{\frac{\beta}{\alpha}} \psi(x) \mathrm{d} x\right]^{\frac{\alpha}{\beta}}
\end{aligned}
$$

Applying this in (30) (and considering (28)) we obtain

$$
B_{7} \lesssim A_{(18)} \lesssim B_{7}+B_{6}
$$

Furthermore, from Proposition 2.1 and integration by parts it follows that $B_{6}+B_{8} \simeq A_{(19)}$. Combining this estimate with (31) and (29), we finally get $B_{5}+B_{6} \simeq B_{6}+B_{7}+B_{8} \simeq A_{(18)}+A_{(19)}$, which we needed to prove.

The next lemma characterizes the embedding $\Lambda^{\gamma}(\omega) \hookrightarrow K^{\alpha, \beta}(\varphi, \psi)$ for $0<\alpha \leq \beta<\infty$ and $\alpha<\gamma<\infty$.

Lemma 3.3. Let $\varphi, \psi, \omega$ be weights. Denote

$$
C_{(32)}:=\sup _{g \in \mathscr{M}} \frac{\left(\int_{0}^{\infty}\left(\int_{x}^{\infty}\left(g^{* *}\right)^{\alpha} \varphi\right)^{\frac{\beta}{\alpha}} \psi(x) \mathrm{d} x\right)^{\frac{1}{\beta}}}{\left(\int_{0}^{\infty}\left(g^{*}\right)^{\gamma} \omega\right)^{\frac{1}{\gamma}}} .
$$

(i) Let $0<\alpha<\gamma \leq \beta<\infty$ and $1<\gamma$. Then $C_{(32)} \simeq A_{(33)}+A_{(34)}+$ $A_{(35)}$, where

$$
\begin{aligned}
A_{(33):}= & \sup _{x>0}\left[\int_{x}^{\infty}\left(\int_{x}^{s} \varphi\right)^{\frac{\gamma}{\gamma-\alpha}} \Omega^{\frac{\gamma}{\alpha-\gamma}}(s) \omega(s) \mathrm{d} s\right]^{\frac{\gamma-\alpha}{\gamma \alpha}} \Psi^{\frac{1}{\beta}}(x) \\
+ & \sup _{x>0} \Omega^{-\frac{1}{\gamma}}(x)\left[\int_{0}^{x}\left(\int_{s}^{x} \varphi\right)^{\frac{\beta}{\alpha}} \psi(s) \mathrm{d} s\right]^{\frac{1}{\beta}}, \\
A_{(34):=} \sup _{x>0}\left[\int_{x}^{\infty}\left(\int_{s}^{\infty} \frac{\varphi(t)}{t^{\alpha}} \mathrm{d} t\right)^{\frac{\alpha}{\gamma-\alpha}} \frac{\varphi(s)}{s^{\alpha}}\right. & \mathrm{d} s]^{\frac{s}{\gamma \alpha}} \Psi^{\frac{1}{\beta}}(x), \\
& \times\left(\int_{0}^{s} \frac{y^{\gamma^{\prime}} \omega(y)}{\Omega^{\gamma^{\prime}}(y)} \mathrm{d} y\right)^{\frac{\alpha(\gamma-1)}{\gamma-\alpha}} \mathrm{d}
\end{aligned}
$$


and

$$
A_{(35)}:=\sup _{x>0}\left[\int_{x}^{\infty}\left(\int_{s}^{\infty} \frac{\varphi(t)}{t^{\alpha}} \mathrm{d} t\right)^{\frac{\beta}{\alpha}} \psi(s) \mathrm{d} s\right]^{\frac{1}{\beta}}\left(\int_{0}^{x} \frac{s^{\gamma^{\prime}} \omega(s)}{\Omega^{\gamma^{\prime}}(s)} \mathrm{d} s\right)^{\frac{1}{\gamma^{\prime}}} .
$$

(ii) Let $0<\alpha<\beta<\gamma<\infty$ and $1<\gamma$. Then $C_{(32)} \simeq A_{(36)}+A_{(37)}+$ $A_{(38)}$, where

$$
\begin{aligned}
& A_{(36)}:= {\left[\int_{0}^{\infty}\left(\int_{x}^{\infty}\left(\int_{x}^{s} \varphi\right)^{\frac{\gamma}{\gamma-\alpha}} \Omega^{\frac{\gamma}{\alpha-\gamma}}(s) \omega(s) \mathrm{d} s\right)^{\frac{\beta(\gamma-\alpha)}{\alpha(\gamma-\beta)}}\right.} \\
&\left.\times \Psi^{\frac{\beta}{\gamma-\beta}}(x) \psi(x) \mathrm{d} x\right]^{\frac{\gamma-\beta}{\gamma \beta}} \\
&+ {\left[\int_{0}^{\infty}\left(\int_{0}^{x}\left(\int_{s}^{x} \varphi\right)^{\frac{\beta}{\alpha}} \psi(s) \mathrm{d} s\right)^{\frac{\beta}{\gamma-\beta}} \int_{0}^{x}\left(\int_{s}^{x} \varphi\right)^{\frac{\beta-\alpha}{\alpha}}\right.} \\
&\left.\times \psi(s) \mathrm{d} s \varphi(x) \Omega^{\frac{\beta}{\beta-\gamma}}(x) \mathrm{d} x\right]^{\frac{\gamma-\beta}{\gamma \beta}},
\end{aligned}
$$

(37) $A_{(37)}:=\left[\int_{0}^{\infty}\left(\int_{x}^{\infty}\left(\int_{s}^{\infty} \frac{\varphi(t)}{t^{\alpha}} \mathrm{d} t\right)^{\frac{\alpha}{\gamma-\alpha}} \frac{\varphi(s)}{s^{\alpha}}\right.\right.$

$$
\left.\left.\times\left(\int_{0}^{s} \frac{y^{\gamma^{\prime}} \omega(y)}{\Omega^{\gamma^{\prime}}(y)} \mathrm{d} y\right)^{\frac{\alpha(\gamma-1)}{\gamma-\alpha}} \mathrm{d} s\right)^{\frac{\beta(\gamma-\alpha)}{\alpha(\gamma-\beta)}} \Psi^{\frac{\beta}{\gamma-\beta}}(x) \psi(x) \mathrm{d} x\right]^{\frac{\gamma-\beta}{\gamma \beta}},
$$

and

$$
\begin{aligned}
A_{(38):=} & {\left[\int_{0}^{\infty}\left(\int_{x}^{\infty}\left(\int_{s}^{\infty} \frac{\varphi(t)}{t^{\alpha}} \mathrm{d} t\right)^{\frac{\beta}{\alpha}} \psi(s) \mathrm{d} s\right)^{\frac{\beta}{\gamma-\beta}}\right.} \\
& \left.\times\left(\int_{x}^{\infty} \frac{\varphi(t)}{t^{\alpha}} \mathrm{d} t\right)^{\frac{\beta}{\alpha}} \psi(x)\left(\int_{0}^{x} \frac{s^{\gamma^{\prime}} \omega(s)}{\Omega^{\gamma^{\prime}}(s)} \mathrm{d} s\right)^{\frac{\beta(\gamma-1)}{\gamma-\beta}} \mathrm{d} x\right]^{\frac{\gamma-\beta}{\gamma \beta}} .
\end{aligned}
$$

(iii) Let $0<\alpha<\gamma \leq \beta<\infty$ and $\gamma \leq 1$. Let $\sigma$ be given by (11). Then $C_{(32)} \simeq A_{(33)}+A_{(39)}+A_{(40)}$, where

$$
A_{(39)}:=\sup _{x>0}\left[\int_{x}^{\infty}\left(\int_{s}^{\infty} \frac{\varphi(t)}{t^{\alpha}} \mathrm{d} t\right)^{\frac{\alpha}{\gamma-\alpha}} \frac{\varphi(s)}{s^{\alpha}} \sigma(s) \mathrm{d} s\right]^{\frac{\gamma-\alpha}{\gamma \alpha}} \Psi^{\frac{1}{\beta}}(x)
$$


and

$$
A_{(40)}:=\sup _{x>0} \sigma^{\frac{\gamma-\alpha}{\gamma \alpha}}(x)\left[\int_{x}^{\infty}\left(\int_{s}^{\infty} \frac{\varphi(t)}{t^{\alpha}} \mathrm{d} t\right)^{\frac{\beta}{\alpha}} \psi(s) \mathrm{d} s\right]^{\frac{1}{\beta}} .
$$

(iv) Let $0<\alpha<\beta<\gamma \leq 1$. Let $\sigma$ be given by (11). Then $C_{(32)} \simeq$ $A_{(36)}+A_{(41)}+A_{(42)}$, where

(41) $A_{(41)}:=\left[\int_{0}^{\infty}\left(\int_{x}^{\infty}\left(\int_{s}^{\infty} \frac{\varphi(t)}{t^{\alpha}} \mathrm{d} t\right)^{\frac{\alpha}{\gamma-\alpha}} \frac{\varphi(s)}{s^{\alpha}} \sigma(s) \mathrm{d} s\right)^{\frac{\beta(\gamma-\alpha)}{\alpha(\gamma-\beta)}} \Psi^{\frac{\beta}{\gamma-\beta}}(x) \psi(x) \mathrm{d} x\right]^{\frac{\gamma-\beta}{\gamma \beta}}$

and

$$
\begin{aligned}
A_{(42)}:=\left[\int_{0}^{\infty}\left(\int_{x}^{\infty}\left(\int_{s}^{\infty} \frac{\varphi(t)}{t^{\alpha}} \mathrm{d} t\right)^{\frac{\beta}{\alpha}} \psi(s) \mathrm{d} s\right)^{\frac{\beta}{\gamma-\beta}}\right. \\
\left.\quad \times\left(\int_{x}^{\infty} \frac{\varphi(y)}{y^{\alpha}} \mathrm{d} y\right)^{\frac{\beta}{\alpha}} \psi(x) \sigma^{\frac{\beta(\gamma-\alpha)}{\alpha(\gamma-\beta)}}(x) \mathrm{d} x\right]^{\frac{\gamma-\beta}{\gamma \beta}} .
\end{aligned}
$$

Proof: The proof is to a great extent analogous to that of Lemma 3.2 but there are some additional steps which we show below.

Let $u$ be defined by (22). If $1>\gamma, L^{p}$-duality and Proposition 2.3 gives

$$
\begin{aligned}
& C_{(12)}=\sup _{h \in \mathscr{M}_{+}} \frac{1}{\left(\int_{0}^{\infty} h^{\frac{\beta}{\beta-\alpha}} \psi^{\frac{\alpha}{\alpha-\beta}}\right)^{\frac{\beta-\alpha}{\beta \alpha}}} \sup _{g \in \mathscr{M}} \frac{\left(\int_{0}^{\infty}\left(g^{* *}(t)\right)^{\alpha} \varphi(t) \int_{0}^{t} h(x) \mathrm{d} x \mathrm{~d} t\right)^{\frac{1}{\alpha}}}{\left(\int_{0}^{\infty}\left(g^{*}\right)^{\gamma} \omega\right)^{\frac{1}{\gamma}}} \\
& \simeq \sup _{h \in \mathscr{M}_{+}} \frac{\left(\int_{0}^{\infty}\left(\int_{0}^{s} \varphi(t) \int_{0}^{t} h(x) \mathrm{d} x \mathrm{~d} t\right)^{\frac{\gamma}{\gamma-\alpha}} \Omega^{\frac{\gamma}{\alpha-\gamma}}(s) \omega(s) \mathrm{d} s\right)^{\frac{\gamma-\alpha}{\gamma \alpha}}}{\left(\int_{0}^{\infty} h^{\frac{\beta}{\beta-\alpha}} \psi^{\frac{\alpha}{\alpha-\beta}}\right)^{\frac{\beta-\alpha}{\beta \alpha}}} \\
& +\sup _{h \in \mathscr{M}_{+}} \frac{\left(\int_{0}^{\infty} \varphi(t) \int_{0}^{t} h(x) \mathrm{d} x \mathrm{~d} t\right)^{\frac{1}{\alpha}}}{\left(\int_{0}^{\infty} h^{\frac{\beta}{\beta-\alpha}} \psi^{\frac{\alpha}{\alpha-\beta}}\right)^{\frac{\beta-\alpha}{\beta \alpha}} \Omega^{\frac{1}{\gamma}}(\infty)} \\
& +\sup _{h \in \mathscr{M}_{+}} \frac{\left(\int_{0}^{\infty}\left(\int_{s}^{\infty} \frac{\varphi(t)}{t^{\alpha}} \int_{0}^{t} h(x) \mathrm{d} x \mathrm{~d} t\right)^{\frac{\alpha}{\gamma-\alpha}} \frac{\varphi(s)}{s^{\alpha}} \int_{0}^{s} h(t) \mathrm{d} t\left(\int_{0}^{s} u(y) \mathrm{d} y\right)^{\frac{\alpha(\gamma-1)}{\gamma-\alpha}} \mathrm{d} s\right)^{\frac{\gamma-\alpha}{\gamma \alpha}}}{\left(\int_{0}^{\infty} h^{\frac{\beta}{\beta-\alpha}} \psi^{\frac{\alpha}{\alpha-\beta}}\right)^{\frac{\beta-\alpha}{\beta \alpha}}} .
\end{aligned}
$$


If $u$ is integrable near the origin, then the term (43) is equivalent to

$$
\sup _{h \in \mathscr{M}_{+}} \frac{\left(\int_{0}^{\infty}\left(\int_{s}^{\infty} \frac{\varphi(t)}{t^{\alpha}} \int_{0}^{t} h(x) \mathrm{d} x \mathrm{~d} t\right)^{\frac{\gamma}{\gamma-\alpha}}\left(\int_{0}^{s} u(y) \mathrm{d} y\right)^{\frac{\gamma(\alpha-1)}{\gamma-\alpha}} u(s) \mathrm{d} s\right)^{\frac{\gamma-\alpha}{\gamma \alpha}}}{\left(\int_{0}^{\infty} h^{\frac{\beta}{\beta-\alpha}} \psi^{\frac{\alpha}{\alpha-\beta}}\right)^{\frac{\beta-\alpha}{\beta \alpha}}} .
$$

(i) Suppose that $u$ is integrable near the origin. As in Lemma 3.2(i), using Hardy inequality, [24, Theorem 1.1] and the dual version of it one shows that $C_{(32)} \simeq A_{(33)}+B_{1}+A_{(35)}$, where

$$
B_{1}:=\sup _{x>0}\left[\int_{x}^{\infty}\left(\int_{s}^{\infty} \frac{\varphi(t)}{t^{\alpha}} \mathrm{d} t\right)^{\frac{\gamma}{\gamma-\alpha}}\left(\int_{0}^{s} \frac{y^{\gamma^{\prime}} \omega(y)}{\Omega^{\gamma^{\prime}}(y)} \mathrm{d} y\right)^{\frac{\gamma(\alpha-1)}{\gamma-\alpha}} \frac{s^{\gamma^{\prime}} \omega(s)}{\Omega^{\gamma^{\prime}}(s)} \mathrm{d} s\right]^{\frac{\gamma-\alpha}{\gamma \alpha}} \Psi^{\frac{1}{\beta}}(x) .
$$

Integration by parts gives $B_{1}+B_{2} \simeq A_{(34)}$ with

$$
B_{2}:=\sup _{x>0}\left(\int_{x}^{\infty} \frac{\varphi(t)}{t^{\alpha}} \mathrm{d} t\right)^{\frac{1}{\alpha}}\left(\int_{0}^{x} \frac{y^{\gamma^{\prime}} \omega(y)}{\Omega^{\gamma^{\prime}}(y)} \mathrm{d} y\right)^{\frac{1}{\gamma^{\prime}}} \Psi^{\frac{1}{\beta}}(x)
$$

Using the proof idea of [13, Lemma 2.2] (a similar problem was also treated in [19, Proposition 3.2]), one checks that $B_{2} \lesssim B_{1}+A_{(35)}$. This implies that $B_{1}+A_{(35)} \simeq A_{(34)}+A_{(35)}$, hence $C_{(32)} \simeq A_{(33)}+A_{(34)}+$ $A_{(35)}$. Finally, we make the following observation, same as in Lemma 3.2. If $u$ is not integrable near the origin, then $C_{(32)}=\infty$ (see (43)) and $A_{(35)}=\infty$. Hence, the equivalence $C_{(32)} \simeq A_{(33)}+B_{1}+A_{(35)}$ holds even without additional assumptions on $u$.

(ii) Analogously to (i) we assume that $u$ is integrable near the origin and get $C_{(32)} \simeq A_{(36)}+B_{3}+A_{(38)}$, where

$$
\begin{array}{r}
B_{3}:=\left[\int_{0}^{\infty}\left(\int_{x}^{\infty}\left(\int_{s}^{\infty} \frac{\varphi(t)}{t^{\alpha}} \mathrm{d} t\right)^{\frac{\gamma}{\gamma-\alpha}}\left(\int_{0}^{s} \frac{y^{\gamma^{\prime}} \omega(y)}{\Omega^{\gamma^{\prime}}(y)} \mathrm{d} y\right)^{\frac{\gamma(\alpha-1)}{\gamma-\alpha}} \frac{s^{\gamma^{\prime}} \omega(s)}{\Omega^{\gamma^{\prime}}(s)} \mathrm{d} s\right)^{\frac{\beta(\gamma-\alpha)}{\alpha(\gamma-\beta)}}\right. \\
\left.\times \Psi^{\frac{\beta}{\gamma-\beta}}(x) \psi(x) \mathrm{d} x\right]^{\frac{\gamma-\beta}{\gamma \beta}}
\end{array}
$$


By integration by parts it follows that $B_{3}+B_{4} \simeq A_{(37)}$, where

$$
B_{4}:=\left[\int_{0}^{\infty}\left(\int_{x}^{\infty} \frac{\varphi(t)}{t^{\alpha}} \mathrm{d} t\right)^{\frac{\beta \gamma}{\alpha(\gamma-\beta)}}\left(\int_{0}^{x} \frac{y^{\gamma^{\prime}} \omega(y)}{\Omega^{\gamma^{\prime}}(y)} \mathrm{d} y\right)^{\frac{\beta(\gamma-1)}{\gamma-\beta}} \Psi^{\frac{\beta}{\gamma-\beta}}(x) \psi(x) \mathrm{d} x\right]^{\frac{\gamma-\beta}{\gamma \beta}}
$$

Following the idea of [14, Theorem 3.1] (cf. [19, Proposition 3.3]) one shows that $B_{4} \lesssim B_{3}+A_{(38)}$. Then $B_{3}+A_{(38)} \simeq A_{(37)}+A_{(38)}$ and thus $C_{(32)} \simeq A_{(36)}+A_{(37)}+A_{(38)}$. The final dropping of the integrability assumption on $u$ is performed in the same way as in (i).

In the remaining part of the proof we will assume that $\gamma \in(0,1]$, which is the case in (iii) and (iv).

(iii) Using the same ideas as in Lemma 3.2(iii), one shows that $C_{(32)} \simeq$ $A_{(33)}+B_{5}+A_{(40)}$, where

$$
B_{5}:=\sup _{x>0}\left[\int_{x}^{\infty}\left(\int_{s}^{\infty} \frac{\varphi(t)}{t^{\alpha}} \mathrm{d} t\right)^{\frac{\gamma}{\gamma-\alpha}} \sigma^{\prime}(s) \mathrm{d} s\right]^{\frac{\gamma-\alpha}{\gamma \alpha}} \Psi^{\frac{1}{\beta}}(x) .
$$

Integration by parts yields

$$
B_{5}+\sup _{x>0}\left(\int_{x}^{\infty} \frac{\varphi(t)}{t^{\alpha}} \mathrm{d} t\right)^{\frac{1}{\alpha}} \sigma^{\frac{\gamma-\alpha}{\gamma \alpha}}(x) \Psi^{\frac{1}{\beta}}(x) \simeq A_{(39)}
$$

hence $B_{5} \lesssim A_{(39)}$. Moreover, it also holds

$$
\sup _{x>0}\left(\int_{x}^{\infty} \frac{\varphi(t)}{t^{\alpha}} \mathrm{d} t\right)^{\frac{1}{\alpha}} \sigma^{\frac{\gamma-\alpha}{\gamma \alpha}}(x) \Psi^{\frac{1}{\beta}}(x) \lesssim B_{5}+A_{(40)},
$$

which is proved by using the same argument from $[\mathbf{1 3}]$ as in (i). Combining the obtained relations, we conclude that $C_{(32)} \simeq A_{(33)}+A_{(39)}+A_{(40)}$.

(iv) In an analogy to Lemma $3.2\left(\right.$ iv) it is proved that $C_{(32)} \simeq A_{(36)}+$ $B_{6}+A_{(42)}$, where

$$
B_{6}:=\left[\int_{0}^{\infty}\left(\int_{x}^{\infty}\left(\int_{s}^{\infty} \frac{\varphi(t)}{t^{\alpha}} \mathrm{d} t\right)^{\frac{\gamma}{\gamma-\alpha}} \sigma^{\prime}(s) \mathrm{d} s\right)^{\frac{\beta(\gamma-\alpha)}{\alpha(\gamma-\beta)}} \Psi^{\frac{\beta}{\gamma-\beta}}(x) \psi(x) \mathrm{d} x\right]^{\frac{\gamma-\beta}{\gamma \beta}}
$$


For any $x>0$, integration by parts gives

$$
\begin{aligned}
\int_{x}^{\infty}\left(\int_{s}^{\infty} \frac{\varphi(t)}{t^{\alpha}} \mathrm{d} t\right)^{\frac{\alpha}{\gamma-\alpha}} \frac{\varphi(s)}{s^{\alpha}} \sigma(s) \mathrm{d} s \simeq \int_{x}^{\infty}\left(\int_{s}^{\infty} \frac{\varphi(t)}{t^{\alpha}} \mathrm{d} t\right)^{\frac{\gamma}{\gamma-\alpha}} \sigma^{\prime}(s) \mathrm{d} s & \\
& +\left(\int_{x}^{\infty} \frac{\varphi(s)}{s^{\alpha}} \mathrm{d} s\right)^{\frac{\gamma}{\gamma-\alpha}} \sigma(x) .
\end{aligned}
$$

Hence, one gets

$$
\begin{aligned}
A_{(41)} \simeq B_{6}+\left[\int_{0}^{\infty}\left(\int_{x}^{\infty} \frac{\varphi(s)}{s^{\alpha}} \mathrm{d} s\right)^{\frac{\gamma \beta}{\alpha(\gamma-\beta)}} \sigma^{\frac{\beta(\gamma-\alpha)}{\alpha(\gamma-\beta)}}(x) \Psi^{\frac{\beta}{\gamma-\beta}}(x) \psi(x) \mathrm{d} x\right]^{\frac{\gamma-\beta}{\gamma \beta}} \\
\simeq B_{6}+\left[\int_{0}^{\infty}\left(\int_{x}^{\infty} \frac{\varphi(s)}{s^{\alpha}} \mathrm{d} s\right)^{\frac{\gamma \beta}{\alpha(\gamma-\beta)}}\left(\int_{0}^{x} \sigma^{\prime}\right)^{\frac{\beta(\gamma-\alpha)}{\alpha(\gamma-\beta)}} \Psi^{\frac{\beta}{\gamma-\beta}}(x) \psi(x) \mathrm{d} x\right]^{\frac{\gamma-\beta}{\gamma \beta}} \\
+\sigma^{\frac{\gamma-\alpha}{\gamma \alpha}}(0+)\left[\int_{0}^{\infty}\left(\int_{x}^{\infty} \frac{\varphi(s)}{s^{\alpha}} \mathrm{d} s\right)^{\frac{\gamma \beta}{\alpha(\gamma-\beta)}} \Psi^{\frac{\beta}{\gamma-\beta}}(x) \psi(x) \mathrm{d} x\right]^{\frac{\gamma-\beta}{\gamma \beta}} \\
=: B_{6}+B_{7}+B_{8} .
\end{aligned}
$$

Using the same argument as in (ii) (based on [14]), we can show that $B_{7} \lesssim B_{6}+A_{(42)}$. Next, since the function $s \mapsto \frac{\varphi(s)}{s^{\alpha}}$ is nonincreasing, we obtain

$$
\left[\int_{0}^{\infty}\left(\int_{x}^{\infty} \frac{\varphi(s)}{s^{\alpha}} \mathrm{d} s\right)^{\frac{\gamma \beta}{\alpha(\gamma-\beta)}} \Psi^{\frac{\beta}{\gamma-\beta}}(x) \psi(x) \mathrm{d} x\right]^{\frac{\alpha(\gamma-\beta)}{\gamma \beta}} \lesssim\left[\int_{0}^{\infty}\left(\int_{x}^{\infty} \frac{\varphi(s)}{s^{\alpha}} \mathrm{d} s\right)^{\frac{\beta}{\alpha}} \psi(x) \mathrm{d} x\right]^{\frac{\alpha}{\beta}}
$$

by using the characterization of the embedding $\Lambda \hookrightarrow \Lambda$ [7, Theorem 3.1]. Thus, since

$$
\sigma^{\frac{\gamma-\alpha}{\gamma \alpha}}(0+)\left[\int_{0}^{\infty}\left(\int_{x}^{\infty} \frac{\varphi(s)}{s^{\alpha}} \mathrm{d} s\right)^{\frac{\beta}{\alpha}} \psi(x) \mathrm{d} x\right]^{\frac{1}{\beta}} \lesssim A_{(42)},
$$

we get the inequality $B_{8} \lesssim A_{(42)}$. Summarizing, we obtained $A_{(41)}+$ $A_{(42)} \simeq B_{6}+A_{(42)}$, hence $C_{(32)} \simeq A_{(36)}+A_{(41)}+A_{(42)}$ and the proof is completed. 
Although $\alpha<\gamma$ was assumed in the above statements, the proof method is not limited to this case. In fact, only the assumption $\alpha \leq \beta$ is crucial for the duality approach. We may hence consider the case $0<\gamma \leq$ $\alpha \leq \beta<\infty$ and characterize the embedding $\Lambda^{\gamma}(\omega) \hookrightarrow J^{\alpha, \beta}(\varphi, \psi)$ using the same technique as before. The proof becomes actually considerably simpler in this case.

Proposition 3.4. Let $\varphi, \psi, \omega$ be weights.

(i) Let $1<\gamma \leq \alpha \leq \beta<\infty$. Then $C_{(12)} \simeq A_{(44)}+A_{(45)}$, where

$$
A_{(44)}:=\sup _{x>0}\left(\int_{0}^{x} \Phi^{\frac{\beta}{\alpha}} \psi\right)^{\frac{1}{\beta}} \Omega^{-\frac{1}{\gamma}}(x)+\sup _{x>0}\left(\int_{x}^{\infty} \psi\right)^{\frac{1}{\beta}} \Phi^{\frac{1}{\alpha}}(x) \Omega^{-\frac{1}{\gamma}}(x)
$$

and

$$
A_{(45)}:=\sup _{x>0}\left(\int_{x}^{\infty}\left(\int_{x}^{t} \frac{\varphi(s)}{s^{\alpha}} \mathrm{d} s\right)^{\frac{\beta}{\alpha}} \psi(t) \mathrm{d} t\right)^{\frac{1}{\beta}}\left(\int_{0}^{x} \frac{t^{\gamma^{\prime}} \omega(t)}{\Omega^{\gamma^{\prime}}(t)} \mathrm{d} t\right)^{\frac{1}{\gamma^{\prime}}} .
$$

(ii) Let $0<\gamma \leq 1$ and $\gamma \leq \alpha \leq \beta<\infty$. Then $C_{(12)} \simeq A_{(44)}+A_{(46)}$, where

$$
A_{(46)}:=\sup _{x>0}\left(\int_{x}^{\infty}\left(\int_{x}^{t} \frac{\varphi(s)}{s^{\alpha}} \mathrm{d} s\right)^{\frac{\beta}{\alpha}} \psi(t) \mathrm{d} t\right)^{\frac{1}{\beta}} x \Omega^{-\frac{1}{\gamma}}(x) .
$$

Proof: Just as in (20) and (21), one has

$$
\begin{aligned}
C_{(12)}= & \sup _{h \in \mathscr{M}_{+}} \frac{1}{\left(\int_{0}^{\infty} h^{\frac{\beta}{\beta-\alpha}} \psi^{\frac{\alpha}{\alpha-\beta}}\right)^{\frac{\beta-\alpha}{\beta \alpha}}} \\
& \times \sup _{g \in \mathscr{M}} \frac{\left(\int_{0}^{\infty}\left(g^{* *}(t)\right)^{\alpha} \varphi(t) \int_{t}^{\infty} h(x) \mathrm{d} x \mathrm{~d} t\right)^{\frac{1}{\alpha}}}{\left(\int_{0}^{\infty}\left(g^{*}\right)^{\gamma} \omega\right)^{\frac{1}{\gamma}}}=: B .
\end{aligned}
$$


Consider the case (i). Then

$$
\begin{aligned}
& B \simeq \sup _{h \in \mathscr{M}_{+}} \sup _{x>0} \frac{\left(\int_{0}^{x} \varphi(t) \int_{t}^{\infty} h(s) \mathrm{d} s \mathrm{~d} t\right)^{\frac{1}{\alpha}} \Omega^{-\frac{1}{\gamma}}(x)}{\left(\int_{0}^{\infty} h^{\frac{\beta}{\beta-\alpha}} \psi^{\frac{\alpha}{\alpha-\beta}}\right)^{\frac{\beta-\alpha}{\beta \alpha}}} \\
& +\sup _{h \in \mathscr{M}_{+}} \sup _{x>0} \frac{\left(\int_{x}^{\infty} \frac{\varphi(t)}{t^{\alpha}} \int_{t}^{\infty} h(s) \mathrm{d} s \mathrm{~d} t\right)^{\frac{1}{\alpha}}\left(\int_{0}^{x} t^{\gamma^{\prime}} \omega(t) \Omega^{-\gamma^{\prime}}(t) \mathrm{d} t\right)^{\frac{1}{\gamma^{\prime}}}}{\left(\int_{0}^{\infty} h^{\frac{\beta}{\beta-\alpha}} \psi^{\frac{\alpha}{\alpha-\beta}}\right)^{\frac{\beta-\alpha}{\beta \alpha}}} \\
& \simeq \sup _{x>0} \sup _{h \in \mathscr{M}_{+}} \frac{\left(\int_{0}^{x} h \Phi\right)^{\frac{1}{\alpha}} \Omega^{-\frac{1}{\gamma}}(x)}{\left(\int_{0}^{\infty} h^{\frac{\beta}{\beta-\alpha}} \psi^{\frac{\alpha}{\alpha-\beta}}\right)^{\frac{\beta-\alpha}{\beta \alpha}}} \\
& +\sup _{x>0} \sup _{h \in \mathscr{M}_{+}} \frac{\left(\int_{x}^{\infty} h\right)^{\frac{1}{\alpha}} \Phi^{\frac{1}{\alpha}}(x) \Omega^{-\frac{1}{\gamma}}(x)}{\left(\int_{0}^{\infty} h^{\frac{\beta}{\beta-\alpha}} \psi^{\frac{\alpha}{\alpha-\beta}}\right)^{\frac{\beta-\alpha}{\beta \alpha}}} \\
& +\sup _{x>0} \sup _{h \in \mathscr{M}_{+}} \frac{\left(\int_{x}^{\infty} h(s) \int_{x}^{s} \frac{\varphi(t)}{t^{\alpha}} \mathrm{d} t \mathrm{~d} s\right)^{\frac{1}{\alpha}}\left(\int_{0}^{x} t^{\gamma^{\prime}} \omega(t) \Omega^{-\gamma^{\prime}}(t) \mathrm{d} t\right)^{\frac{1}{\gamma^{\prime}}}}{\left(\int_{0}^{\infty} h^{\frac{\beta}{\beta-\alpha}} \psi^{\frac{\alpha}{\alpha-\beta}}\right)^{\frac{\beta-\alpha}{\beta \alpha}}} \\
& \text { (49) }=A_{(44)}+A_{(45)} \text {. }
\end{aligned}
$$

Step (47) follows by [7, Theorem 4.1(i)], step (48) by Fubini theorem and changing the order of the suprema, and (49) is due to $L^{p}$-duality.

Case (ii) is proved analogously, using [7, Theorem 4.1(ii)] to estimate $B$.

Proving an analogous proposition concerning the embedding $\Lambda^{\gamma}(\omega) \hookrightarrow$ $K^{\alpha, \beta}(\varphi, \psi), 0<\gamma \leq \alpha \leq \beta<\infty$, is left to an interested reader.

\section{Bilinear Hardy inequality}

At this point we have all the preliminary results needed to characterize the validity of the Hardy-type inequality (4) or, in other words, to provide equivalent estimates on $C_{(1)}$. The form of the results depends on the values of the exponents $p_{1}, p_{2}$, and $q$ and their mutual relation. 
In fact, in this three-parameter setting, 23 different cases are possible and need separate treatment. For a better orientation, we present all the possible settings in the table below with references to the theorem in which each particular case is presented. Note that in some cases the roles of $p_{1}$ and $p_{2}$ may be switched in the corresponding theorem, compared with the entry in the table.

\begin{tabular}{|c|c|c|c|c|}
\hline \multicolumn{4}{|c|}{ Configuration of the exponents } & Theorem \\
\hline \multirow{9}{*}{$0<p_{1}, p_{2} \leq q$} & \multirow{2}{*}{$0<p_{1}, p_{2} \leq 1$} & \multicolumn{2}{|r|}{$q<\infty$} & $4.2(\mathrm{i})$ \\
\hline & & \multicolumn{2}{|r|}{$q=\infty$} & 4.4(i) \\
\hline & \multirow{3}{*}{$0<p_{1} \leq 1<p_{2}$} & \multicolumn{2}{|r|}{$q<\infty$} & 4.1 (ii) \\
\hline & & \multirow{2}{*}{$q=\infty$} & $p_{2}<\infty$ & $4.4(\mathrm{ii})$ \\
\hline & & & $p_{2}=\infty$ & 4.4(iii) \\
\hline & \multirow{4}{*}{$1<p_{1}, p_{2}$} & \multicolumn{2}{|r|}{$q<\infty$} & $4.1(\mathrm{i})$ \\
\hline & & \multirow{3}{*}{$q=\infty$} & $p_{1}, p_{2}<\infty$ & $4.4(\mathrm{iv})$ \\
\hline & & & $p_{1}<p_{2}=\infty$ & $4.4(\mathrm{v})$ \\
\hline & & & $p_{1}=p_{2}=\infty$ & $4.4(\mathrm{vi})$ \\
\hline \multirow{5}{*}{$0<p_{1} \leq q<p_{2}$} & \multirow{3}{*}{$0<p_{1} \leq 1$} & \multicolumn{2}{|r|}{$p_{2} \leq 1$} & $4.2(\mathrm{iii})$ \\
\hline & & \multicolumn{2}{|c|}{$1<p_{2}<\infty$} & $4.2(\mathrm{ii})$ \\
\hline & & \multicolumn{2}{|r|}{$p_{2}=\infty$} & $4.5(\mathrm{ii})$ \\
\hline & \multirow{2}{*}{$1<p_{1}$} & \multicolumn{2}{|r|}{$p_{2}<\infty$} & 4.1(iii) \\
\hline & & \multicolumn{2}{|c|}{$p_{2}=\infty$} & $4.5(\mathrm{i})$ \\
\hline \multirow{9}{*}{$0<q<p_{1}, p_{2}$} & \multirow{2}{*}{$0<p_{1}, p_{2} \leq 1$} & \multicolumn{2}{|c|}{$1 / q \geq 1 / p_{1}+1 / p_{2}$} & $4.3(\mathrm{v})$ \\
\hline & & \multicolumn{2}{|c|}{$1 / q>1 / p_{1}+1 / p_{2}$} & $4.3(\mathrm{vi})$ \\
\hline & \multirow{3}{*}{$0<p_{2} \leq 1<p_{1}$} & \multirow{2}{*}{$p_{1}<\infty$} & $1 / q \geq 1 / p_{1}+1 / p_{2}$ & 4.3(iii) \\
\hline & & & $1 / q>1 / p_{1}+1 / p_{2}$ & 4.3 (iv) \\
\hline & & \multicolumn{2}{|c|}{$p_{1}=\infty$} & 4.5 (iv) \\
\hline & \multirow{4}{*}{$1<p_{1}, p_{2}$} & \multirow{2}{*}{$p_{1}, p_{2}<\infty$} & $1 / q \geq 1 / p_{1}+1 / p_{2}$ & $4.3(\mathrm{i})$ \\
\hline & & & $1 / q>1 / p_{1}+1 / p_{2}$ & $4.3(\mathrm{ii})$ \\
\hline & & \multicolumn{2}{|c|}{$p_{1}<p_{2}=\infty$} & 4.5(iii) \\
\hline & & \multicolumn{2}{|c|}{$p_{1}=p_{2}=\infty$} & $4.5(\mathrm{v})$ \\
\hline
\end{tabular}

Let us now present and prove the results. We start with the configurations in which only the "classical" spaces appear, i.e. those where all the exponents are finite. First such case is $1<p_{1} \leq q<\infty$. 
Theorem 4.1. Let $v_{1}, v_{2}, w$ be weights.

(i) Let $1<p_{1}, p_{2} \leq q$. Then $C_{(1)} \simeq A_{(50)}+A_{(51)}^{1,2}+A_{(51)}^{2,1}+A_{(52)}$, where

$$
A_{(51)}^{i, j}:=\sup _{0<t<x<\infty}\left(\int_{t}^{x} \frac{w(s)}{s^{q}} \mathrm{~d} s\right)^{\frac{1}{q}} V_{i}^{-\frac{1}{p_{i}}}(x)\left(\int_{0}^{t} \frac{s^{p_{j}^{\prime}} v_{j}(s)}{V_{j}^{p_{j}^{\prime}}(s)} \mathrm{d} s\right)^{\frac{1}{p_{j}^{\prime}}},
$$

and

$$
A_{(52)}:=\sup _{t>0}\left(\int_{t}^{\infty} \frac{w(s)}{s^{2 q}} \mathrm{~d} s\right)^{\frac{1}{q}}\left(\int_{0}^{t} \frac{s^{p_{1}^{\prime}} v_{1}(s)}{V_{1}^{p_{1}^{\prime}}(s)} \mathrm{d} s\right)^{\frac{1}{p_{1}^{\prime}}}\left(\int_{0}^{t} \frac{s^{p_{2}^{\prime}} v_{2}(s)}{V_{2}^{p_{2}^{\prime}}(s)} \mathrm{d} s\right)^{\frac{1}{p_{2}^{\prime}}} .
$$

(ii) Let $0<p_{2} \leq 1<p_{1} \leq q$. Then $C_{(1)} \simeq A_{(50)}+A_{(52)}^{1,2}+A_{(51)}^{2,1}+A_{(53)}$, where

$$
A_{(52)}^{i, j}:=\sup _{0<t<x<\infty}\left(\int_{t}^{x} \frac{w(s)}{s^{q}} \mathrm{~d} s\right)^{\frac{1}{q}} V_{i}^{-\frac{1}{p_{i}}}(x) t V_{j}^{-\frac{1}{p_{j}}}(t)
$$

and

$$
A_{(53)}:=\sup _{t>0}\left(\int_{t}^{\infty} \frac{w(s)}{s^{2 q}} \mathrm{~d} s\right)^{\frac{1}{q}}\left(\int_{0}^{t} \frac{s^{p_{1}^{\prime}} v_{1}(s)}{V_{1}^{p_{1}^{\prime}}(s)} \mathrm{d} s\right)^{\frac{1}{p_{1}^{\prime}}} t V_{2}^{-\frac{1}{p_{2}}}(t) .
$$

(iii) Let $1<p_{1} \leq q<p_{2}<\infty$. Define $r_{2}:=\frac{p_{2} q}{p_{2}-q}$. Then $C_{(1)} \simeq$ $A_{(54)}+A_{(55)}+A_{(56)}$, where

$$
A_{(54)}:=\sup _{x>0} V_{1}^{-\frac{1}{p_{1}}}(x)\left(\int_{0}^{x} W^{\frac{r_{2}}{p_{2}}}(t) w(t) V_{2}^{-\frac{r_{2}}{p_{2}}}(t) \mathrm{d} t\right)^{\frac{1}{r_{2}}},
$$

(55) $A_{(55)}:=\sup _{x>0} V_{1}^{-\frac{1}{p_{1}}}(x)\left[\int_{0}^{x}\left(\int_{t}^{x} \frac{w(s)}{s^{q}} \mathrm{~d} s\right)^{\frac{r_{2}}{p_{2}}} \frac{w(t)}{t^{q}}\left(\int_{0}^{t} \frac{v_{2}(s) s^{p_{2}^{\prime}}}{V_{2}^{p_{2}^{\prime}}(s)} \mathrm{d} s\right)^{\frac{r_{2}}{p_{2}^{\prime}}} \mathrm{d} t\right]^{\frac{1}{r_{2}}}$, 
and

(56) $A_{(56)}:=\sup _{x>0}\left(\int_{0}^{x} \frac{v_{1}(s) s^{p_{1}^{\prime}}}{V_{1}^{p_{1}^{\prime}}(s)} \mathrm{d} s\right)^{\frac{1}{p_{1}^{\prime}}}\left[\int_{x}^{\infty}\left(\int_{x}^{t} \frac{w(s)}{s^{q}} \mathrm{~d} s\right)^{\frac{r_{2}}{p_{2}}} \frac{w(t)}{t^{q}} V_{2}^{-\frac{r_{2}}{p_{2}}}(t) \mathrm{d} t\right]^{\frac{1}{r_{2}}}$

$$
\begin{aligned}
+\sup _{x>0}\left(\int_{0}^{x} \frac{v_{1}(s) s^{p_{1}^{\prime}}}{V_{1}^{p_{1}^{\prime}}(s)} \mathrm{d} s\right)^{\frac{1}{p_{1}^{\prime}}} & {\left[\int_{x}^{\infty}\left(\int_{t}^{\infty} \frac{w(s)}{s^{2 q}} \mathrm{~d} s\right)^{\frac{r_{2}}{p_{2}}} \frac{w(t)}{t^{2 q}}\right.} \\
& \left.\times\left(\int_{0}^{t} \frac{v_{2}(s) s^{p_{2}^{\prime}}}{V_{2}^{p_{2}^{\prime}}(s)} \mathrm{d} s\right)^{\frac{r_{2}}{p_{2}^{\prime}}} \mathrm{d} t\right]^{\frac{1}{r_{2}}} .
\end{aligned}
$$

Proof: Since $1<p_{1} \leq q<\infty$, by [7, Theorem 4.1(i)], we get

$$
\begin{aligned}
C_{(1)} \simeq & \sup _{g \in \Lambda^{p_{2}}\left(v_{2}\right)} \sup _{x>0}\left(\int_{0}^{x}\left(g^{* *}\right)^{q} w\right)^{\frac{1}{q}} V_{1}^{-\frac{1}{p_{1}}}(x)\|g\|_{\Lambda^{p_{2}}\left(v_{2}\right)}^{-1} \\
& +\sup _{g \in \Lambda^{p_{2}}\left(v_{2}\right)} \sup _{x>0}\left(\int_{x}^{\infty} \frac{\left(g^{* *}(s)\right)^{q} w(s)}{s^{q}} \mathrm{~d} s\right)^{\frac{1}{q}}\left(\int_{0}^{x} \frac{s^{p_{1}^{\prime}} v_{1}(s)}{V_{1}^{p_{1}^{\prime}}(s)} \mathrm{d} s\right)^{\frac{1}{p_{1}^{\prime}}}\|g\|_{\Lambda^{p_{2}}\left(v_{2}\right)}^{-1} \\
= & \sup _{x>0} V_{1}^{-\frac{1}{p_{1}}}(x) \sup _{g \in \Lambda^{p_{2}\left(v_{2}\right)}}\left(\int_{0}^{x}\left(g^{* *}\right)^{q} w\right)^{\frac{1}{q}}\|g\|_{\Lambda^{p_{2}\left(v_{2}\right)}}^{-1} \\
& +\sup _{x>0}\left(\int_{0}^{x} \frac{s^{p_{1}^{\prime}} v_{1}(s)}{V_{1}^{p_{1}^{\prime}}(s)} \mathrm{d} s\right)^{\frac{1}{p_{1}^{\prime}}} \sup _{g \in \Lambda^{p_{2}\left(v_{2}\right)}}\left(\int_{x}^{\infty} \frac{\left(g^{* *}(s)\right)^{q} w(s)}{s^{q}} \mathrm{~d} s\right)^{\frac{1}{q}}\|g\|_{\Lambda^{p_{2}}\left(v_{2}\right)}^{-1} \\
= & \sup _{x>0} V_{1}^{-\frac{1}{p_{1}}}(x)\|\operatorname{Id}\|_{\Lambda^{p_{2}}\left(v_{2}\right) \rightarrow \Gamma^{q}\left(w \chi_{[0, x]}\right)} \\
& +\sup _{x>0}\left(\int_{0}^{x} \frac{s^{p_{1}^{\prime}} v_{1}(s)}{V_{1}^{p_{1}^{\prime}}(s)} \mathrm{d} s\right)^{\frac{1}{p_{1}^{\prime}}}\|\operatorname{Id}\|_{\Lambda^{p_{2}}\left(v_{2}\right) \rightarrow \Gamma^{q}\left(s \mapsto w(s) s^{-q} \chi_{[x, \infty)}(s)\right)} \\
= & : B_{1}+B_{2} .
\end{aligned}
$$

Now we separate the different cases. In (i), [7, Theorem 4.1(i)] yields $B_{1}+B_{2} \simeq A_{(50)}+A_{(51)}^{1,2}+A_{(51)}^{2,1}+A_{(52)}$. In (ii), [7, Theorem 4.1(ii)] gives that $B_{1} \simeq A_{(50)}+A_{(52)}^{1,2}$ and $B_{2} \simeq A_{(51)}^{2,1}+A_{(53)}$. Finally, in (iii), Proposition 2.3 yields $B_{1}+B_{2} \simeq A_{(54)}+A_{(55)}+A_{(56)}$.

Now we consider the case $0<p_{1} \leq 1, p_{1} \leq q$. 
Theorem 4.2. Let $v_{1}, v_{2}, w$ be weights.

(i) Let $0<p_{1}, p_{2} \leq 1$ and $0<p_{1}, p_{2} \leq q$. Then $C_{(1)} \simeq A_{(50)}+A_{(52)}^{1,2}+$ $A_{(52)}^{2,1}+A_{(57)}^{1,2}+A_{(57)}^{2,1}$, where

$$
A_{(57)}^{i, j}:=\sup _{0<x<t<\infty}\left(\int_{t}^{\infty} \frac{w(s)}{s^{2 q}} \mathrm{~d} s\right)^{\frac{1}{q}} t V_{i}^{-\frac{1}{p_{i}}}(t) x V_{j}^{-\frac{1}{p_{j}}}(x) .
$$

(ii) Let $0<p_{1} \leq 1<p_{2}<\infty$ and $p_{1} \leq q<p_{2}$. Then $C_{(1)} \simeq A_{(54)}+$ $A_{(55)}+A_{(58)}+A_{(59)}$, where

$$
A_{(58)}:=\sup _{x>0} x V_{1}^{-\frac{1}{p_{1}}}(x)\left[\int_{x}^{\infty}\left(\int_{x}^{t} \frac{w(s)}{s^{q}} \mathrm{~d} s\right)^{\frac{r_{2}}{p_{2}}} \frac{w(t)}{t^{q}} V_{2}^{-\frac{r_{2}}{p_{2}}}(t) \mathrm{d} t\right]^{\frac{1}{r_{2}}}
$$

and

(59) $\quad A_{(59)}:=\sup _{x>0} x V_{1}^{-\frac{1}{p_{1}}}(x)\left[\int_{x}^{\infty}\left(\int_{t}^{\infty} \frac{w(s)}{s^{2 q}} \mathrm{~d} s\right)^{\frac{r_{2}}{p_{2}}} \frac{w(t)}{t^{2 q}}\right.$

$$
\left.\times\left(\int_{0}^{t} \frac{s^{p_{2}^{\prime}} v_{2}(s)}{V_{2}^{p_{2}^{\prime}}(s)} \mathrm{d} s\right)^{\frac{r_{2}}{p_{2}^{\prime}}} \mathrm{d} t\right]^{\frac{1}{r_{2}}}
$$

(iii) Let $0<p_{1} \leq q<p_{2} \leq 1$. Then $C_{(1)} \simeq A_{(54)}+A_{(58)}+A_{(60)}$, where

$$
\text { (60) } \begin{aligned}
A_{(60)}:= & \sup _{x>0} V_{1}^{-\frac{1}{p_{1}}}(x)\left[\int_{0}^{x}\left(\int_{t}^{x} \frac{w(s)}{s^{q}} \mathrm{~d} s\right)^{\frac{r_{2}}{p_{2}}} \frac{w(t)}{t^{q}} \sup _{s \in(0, t)} \frac{s^{r_{2}}}{V_{2}^{\frac{r_{2}}{p_{2}}}(s)} \mathrm{d} t\right]^{\frac{1}{r_{2}}} \\
& +\sup _{x>0} x V_{1}^{-\frac{1}{p_{1}}}(x)\left[\int_{x}^{\infty}\left(\int_{t}^{\infty} \frac{w(s)}{s^{2 q}} \mathrm{~d} s\right)^{\frac{r_{2}}{p_{2}}} \frac{w(t)}{t^{2 q}} \sup _{s \in(0, t)} \frac{s^{r_{2}}}{V_{2}^{\frac{r_{2}}{p_{2}}}(s)} \mathrm{d} t\right]^{\frac{1}{r_{2}}} .
\end{aligned}
$$


Proof: Similarly as in Theorem 4.1, by [7, Theorem 4.1(ii)] (since $0<$ $\left.p_{1} \leq 1, p_{1} \leq q<\infty\right)$ we obtain

$$
\begin{aligned}
C_{(1)} \simeq & \sup _{g \in \Lambda^{p_{2}}\left(v_{2}\right)} \sup _{x>0}\left(\int_{0}^{x}\left(g^{* *}\right)^{q} w\right)^{\frac{1}{q}} V_{1}^{-\frac{1}{p_{1}}}(x)\|g\|_{\Lambda^{p_{2}}\left(v_{2}\right)}^{-1} \\
& +\sup _{g \in \Lambda^{p_{2}}\left(v_{2}\right)} \sup _{x>0}\left(\int_{x}^{\infty} \frac{\left(g^{* *}(s)\right)^{q} w(s)}{s^{q}} \mathrm{~d} s\right)^{\frac{1}{q}} x V_{1}^{-\frac{1}{p_{1}}}(x)\|g\|_{\Lambda^{p_{2}}\left(v_{2}\right)}^{-1} \\
= & \sup _{x>0} V_{1}^{-\frac{1}{p_{1}}}(x)\|\operatorname{Id}\|_{\Lambda^{p_{2}}\left(v_{2}\right) \rightarrow \Gamma^{q}\left(w \chi_{[0, x]}\right)} \\
& +\sup _{x>0} x V_{1}^{-\frac{1}{p_{1}}}(x)\|\operatorname{Id}\|_{\Lambda^{p_{2}}\left(v_{2}\right) \rightarrow \Gamma^{q}\left(s \mapsto w(s) s^{-q} \chi_{[x, \infty)}(s)\right)} \\
= & : B_{1}+B_{2} .
\end{aligned}
$$

In (i), by [7, Theorem 4.1(ii)], we have $B_{1}+B_{2} \simeq A_{(50)}+A_{(52)}^{1,2}+A_{(52)}^{2,1}+$ $A_{(57)}^{1,2}+A_{(57)}^{2,1}$. In (ii) it is $B_{1}+B_{2} \simeq A_{(54)}+A_{(55)}+A_{(58)}+A_{(59)}$ by Proposition 2.3 and finally in (iii) one gets $B_{1}+B_{2} \simeq A_{(54)}+A_{(58)}+A_{(60)}$ by $[\mathbf{6}$, Theorem 3.1].

We continue with the case $0<q<p_{1}, p_{2}<\infty$. This case is usually the most complicated one, especially if $p_{1}, p_{2} \leq 1$. Recall that if $q \in$ $(0,1) \cup(1, \infty)$, then $q^{\prime}:=\frac{q}{q-1}$, while if $q=1$, then $q^{\prime}:=\infty$.

Theorem 4.3. Let $v_{1}, v_{2}, w$ be weights. Let $0<q<p_{1}, p_{2}<\infty$. Define $r_{i}:=\frac{p_{i} q}{p_{i}-q}, i \in\{1,2\}$, and $R:=\frac{p_{1} p_{2} q}{p_{1} p_{2}-p_{1} q-p_{2} q}$.

(i) Let $1<p_{1}, p_{2}$ and $\frac{1}{q} \leq \frac{1}{p_{1}}+\frac{1}{p_{2}}$. Then $C_{(1)} \simeq A_{(61)}^{1,2}+A_{(61)}^{2,1}+A_{(62)}^{1,2}+$ $A_{(62)}^{2,1}+A_{(63)}^{1,2}+A_{(63)}^{2,1}+A_{(64)}^{1,2}+A_{(64)}^{2,1}$, where

$$
A_{(61)}^{i, j}:=\sup _{x>0}\left(\int_{0}^{x} W^{\frac{r_{i}}{p_{i}}} w V_{i}^{-\frac{r_{i}}{p_{i}}}\right)^{\frac{1}{r_{i}}} V_{j}^{-\frac{1}{p_{j}}}(x),
$$

(62) $A_{(62)}^{i, j}:=\sup _{x>0}\left[\int_{x}^{\infty}\left(\int_{x}^{s} \frac{w(t)}{t^{q}} \mathrm{~d} t\right)^{\frac{r_{j}}{q}} V_{j}^{-\frac{r_{j}}{q}}(s) v_{j}(s) \mathrm{d} s\right]^{\frac{1}{r_{j}}}\left(\int_{0}^{x} \frac{t^{p_{i}^{\prime}} v_{i}(t)}{V_{i}^{p_{i}^{\prime}}(t)} \mathrm{d} t\right)^{\frac{1}{p_{i}^{\prime}}}$, 
(63) $A_{(63)}^{i, j}:=\sup _{x>0}\left[\int_{0}^{x}\left(\int_{s}^{x} \frac{w(t)}{t^{q}} \mathrm{~d} t\right)^{\frac{r_{i}}{p_{i}}} \frac{w(s)}{s^{q}}\left(\int_{0}^{s} \frac{t^{p_{i}^{\prime}} v_{i}(t)}{V_{i}^{p_{i}^{\prime}}(t)} \mathrm{d} t\right)^{\frac{r_{i}}{p_{i}^{\prime}}} \mathrm{d} s\right]^{\frac{1}{r_{i}}} V_{j}^{-\frac{1}{p_{j}}}(x)$,

and

$$
\begin{array}{r}
A_{(64)}^{i, j}:=\sup _{x>0}\left[\int_{x}^{\infty}\left(\int_{s}^{\infty} \frac{w(t)}{t^{2 q}} \mathrm{~d} t\right)^{\frac{r_{j}}{p_{j}}} \frac{w(s)}{s^{2 q}}\left(\int_{0}^{s} \frac{t^{p_{j}^{\prime}} v_{j}(t)}{V_{j}^{p_{j}^{\prime}}(t)} \mathrm{d} t\right)^{\frac{r_{j}}{p_{j}^{\prime}}} \mathrm{d} s\right]^{\frac{1}{r_{j}}} \\
\times\left(\int_{0}^{x} \frac{t^{p_{i}^{\prime}} v_{i}(t)}{V_{i}^{p_{i}^{\prime}}(t)} \mathrm{d} t\right)^{\frac{1}{p_{i}^{\prime}}} .
\end{array}
$$

(ii) Let $1<p_{1}, p_{2}$ and $\frac{1}{q}>\frac{1}{p_{1}}+\frac{1}{p_{2}}$. Then $C_{(1)} \simeq A_{(65)}^{1,2}+A_{(65)}^{2,1}+A_{(66)}^{1,2}+$ $A_{(66)}^{2,1}+A_{(67)}^{1,2}+A_{(67)}^{2,1}$, where

$$
\begin{aligned}
A_{(65)}^{i, j}:= & {\left[\int_{0}^{\infty}\left(\int_{0}^{x} W^{\frac{r_{j}}{p_{j}}} w V_{j}^{-\frac{r_{j}}{p_{j}}}\right)^{\frac{r_{j}}{p_{i}-r_{j}}}\right.} \\
& \left.\quad \times W^{\frac{r_{j}}{p_{j}}}(x) w(x) V_{j}^{-\frac{r_{j}}{p_{j}}}(x) V_{i}^{\frac{r_{j}}{r_{j}-p_{i}}}(x) \mathrm{d} x\right]^{\frac{1}{R}},
\end{aligned}
$$

(66)

$$
\begin{aligned}
A_{(66)}^{i, j}:= & {\left[\int_{0}^{\infty}\left(\int_{x}^{\infty}\left(\int_{x}^{s} \frac{w(t)}{t^{q}} \mathrm{~d} t\right)^{\frac{r_{j}}{q}} V_{j}^{-\frac{r_{j}}{q}}(s) v_{j}(s) \mathrm{d} s\right)^{\frac{r_{j}}{p_{i}-r_{j}}} \frac{w(x)}{x^{q}}\right.} \\
& \left.\times \int_{x}^{\infty}\left(\int_{x}^{s} \frac{w(t)}{t^{q}} \mathrm{~d} t\right)^{\frac{r_{j}}{p_{j}}} V_{j}^{-\frac{r_{j}}{q}}(s) v_{j}(s) \mathrm{d} s\left(\int_{0}^{x} \frac{s^{p_{i}^{\prime}} v_{i}(s)}{V^{p_{i}^{\prime}}(s)} \mathrm{d} s\right)^{\frac{p_{i}\left(r_{j}-1\right)}{p_{i}-r_{j}}} \mathrm{~d} x\right]^{\frac{1}{R}} \\
+ & {\left[\int_{0}^{\infty}\left(\int_{0}^{x}\left(\int_{s}^{x} \frac{w(t)}{t^{q}} \mathrm{~d} t\right)^{\frac{r_{i}}{p_{i}}} \frac{w(s)}{s^{q}}\left(\int_{0}^{s} \frac{y^{p_{i}^{\prime}} v_{i}(y)}{V_{i}^{p_{i}^{\prime}}(y)} \mathrm{d} y\right)^{\frac{r_{i}}{p_{i}^{\prime}}} \mathrm{d} s\right)^{\frac{r_{i}}{p_{j}-r_{i}}} \frac{w(x)}{x^{q}}\right.} \\
& \left.\times \int_{0}^{x}\left(\int_{s}^{x} \frac{w(t)}{t^{q}} \mathrm{~d} t\right)^{\frac{r_{i}-p_{i}}{p_{i}}} \frac{w(s)}{s^{q}}\left(\int_{0}^{s} \frac{y^{p_{i}^{\prime}} v_{i}(y)}{V_{i}^{p_{i}^{\prime}}(y)} \mathrm{d} y\right)^{\frac{r_{i}}{p_{i}^{\prime}}} \mathrm{d} s V_{j}^{\frac{r_{i}}{r_{i}-p_{j}}}(x) \mathrm{d} x\right]^{\frac{1}{R}},
\end{aligned}
$$


and

$$
\begin{aligned}
A_{(67)}^{i, j}:= & {\left[\int_{0}^{\infty}\left(\int_{x}^{\infty}\left(\int_{s}^{\infty} \frac{w(t)}{t^{2 q}} \mathrm{~d} t\right)^{\frac{r_{i}}{p_{i}}} \frac{w(s)}{s^{2 q}}\left(\int_{0}^{s} \frac{y^{p_{i}^{\prime}} v_{i}(y)}{V_{i}^{p_{i}^{\prime}}(y)} \mathrm{d} y\right)^{\frac{r_{i}}{p_{i}^{\prime}}} \mathrm{d} s\right)^{\frac{r_{i}}{p_{j}-r_{i}}}\right.} \\
& \times\left(\int_{x}^{\infty} \frac{w(t)}{t^{2 q}} \mathrm{~d} t\right)^{\frac{r_{i}}{p_{i}}} \frac{w(x)}{x^{2 q}}\left(\int_{0}^{x} \frac{y^{p_{i}^{\prime}} v_{i}(y)}{V_{i}^{p_{i}^{\prime}}(y)} \mathrm{d} y\right)^{\frac{r_{i}}{p_{i}^{\prime}}} \\
& \left.\times\left(\int_{0}^{x} \frac{s^{p_{j}^{\prime}} v_{j}(s)}{V_{j}^{p_{j}^{\prime}}(s)} \mathrm{d} s\right)^{\frac{r_{i}\left(p_{j}-1\right)}{p_{j}-r_{i}}} \mathrm{~d} x\right]^{\frac{1}{R}} .
\end{aligned}
$$

(iii) Let $p_{2} \leq 1<p_{1}$ and $\frac{1}{q} \leq \frac{1}{p_{1}}+\frac{1}{p_{2}}$. Then $C_{(1)} \simeq A_{(61)}^{1,2}+A_{(61)}^{2,1}+$ $A_{(62)}^{1,2}+A_{(63)}^{1,2}+A_{(68)}$, where

(68) $A_{(68)}:=\sup _{x>0}\left[\int_{0}^{x}\left(\int_{s}^{x} \frac{w(t)}{t^{q}} \mathrm{~d} t\right)^{\frac{r_{2}}{p_{2}}} \frac{w(s)}{s^{q}} \sup _{y \in(0, s)} y^{r_{2}} V_{2}^{-\frac{r_{2}}{p_{2}}}(y)\right]^{\frac{1}{r_{2}}} V_{1}^{-\frac{1}{p_{1}}}(x)$

$$
\begin{aligned}
+\sup _{x>0}\left[\int_{x}^{\infty}\left(\int_{x}^{s} \frac{w(t)}{t^{q}} \mathrm{~d} t\right)^{\frac{r_{1}}{q}} V_{1}^{-\frac{r_{1}}{q}}(s) v_{1}(s) \mathrm{d} s\right]^{\frac{1}{r_{1}}} x V_{2}^{-\frac{1}{p_{2}}}(x) \\
+\sup _{x>0}\left[\int_{x}^{\infty}\left(\int_{s}^{\infty} \frac{w(t)}{t^{2 q}} \mathrm{~d} t\right)^{\frac{r_{2}}{p_{2}}} \frac{w(s)}{s^{2 q}} \sup _{y \in(0, s)} y^{r_{2}} V_{2}^{-\frac{r_{2}}{p_{2}}}(y) \mathrm{d} s\right]^{\frac{1}{r_{2}}} \\
+\sup _{x>0}\left[\int_{x}^{\infty}\left(\int_{s}^{\infty} \frac{w(t)}{t^{2 q}} \mathrm{~d} t\right)^{\frac{r_{1}}{p_{1}}} \frac{w^{p_{1}^{\prime}} v_{1}(s)}{v_{1}^{p_{1}^{\prime}}(s)} \mathrm{d} s\right)^{\frac{1}{p_{1}^{\prime}}} \\
\left.\times\left(\int_{0}^{s} \frac{y^{p_{1}^{\prime}} v_{1}(y)}{V_{1}^{p_{1}^{\prime}}(y)} \mathrm{d} y\right)^{\frac{r_{1}}{p_{1}^{\prime}}} \mathrm{d} s\right]^{\frac{1}{r_{1}}} x V_{2}^{-\frac{1}{p_{2}}}(x) .
\end{aligned}
$$


(iv) Let $p_{2} \leq 1<p_{1}$ and $\frac{1}{q}>\frac{1}{p_{1}}+\frac{1}{p_{2}}$. Then $C_{(1)} \simeq A_{(65)}^{1,2}+A_{(65)}^{2,1}+$ $A_{(66)}^{1,2}+A_{(69)}$, where

(69)

$$
\begin{aligned}
& A_{(69)}:=\left[\int_{0}^{\infty}\left(\int_{0}^{x}\left(\int_{s}^{x} \frac{w(t)}{t^{q}} \mathrm{~d} t\right)^{\frac{r_{2}}{p_{2}}} \frac{w(s)}{s^{q}} \sup _{y \in(0, s)} y^{r_{2}} V_{2}^{-\frac{r_{2}}{p_{2}}}(y) \mathrm{d} s\right)^{\frac{r_{2}}{p_{1}-r_{2}}}\right. \\
& \left.\times \frac{w(x)}{x^{q}} \int_{0}^{x}\left(\int_{s}^{x} \frac{w(t)}{t^{q}} \mathrm{~d} t\right)^{\frac{r_{2}-p_{2}}{p_{2}}} \frac{w(s)}{s^{q}} \sup _{y \in(0, s)} y^{r_{2}} V_{2}^{-\frac{r_{2}}{p_{2}}}(y) \mathrm{d} s V_{1}^{\frac{r_{2}}{r_{2}-p_{1}}}(x) \mathrm{d} x\right]^{\frac{1}{R}} \\
& +\left[\int_{0}^{\infty}\left(\int_{x}^{\infty}\left(\int_{x}^{s} \frac{w(t)}{t^{q}} \mathrm{~d} t\right)^{\frac{r_{1}}{q}} V_{1}^{-\frac{r_{1}}{q}}(s) v_{1}(s) \mathrm{d} s\right)^{\frac{r_{1}}{p_{2}-r_{1}}}\right. \\
& \left.\times \frac{w(x)}{x^{q}} \int_{x}^{\infty}\left(\int_{x}^{s} \frac{w(t)}{t^{q}} \mathrm{~d} t\right)^{\frac{r_{1}}{p_{1}}} V_{1}^{-\frac{r_{1}}{q}}(s) v_{1}(s) \mathrm{d} s \sup _{y \in(0, x)} y^{R} V_{2}^{\frac{r_{1}}{r_{1}-p_{2}}}(y)\right]^{\frac{1}{R}} \\
& +\left[\int_{0}^{\infty}\left(\int_{x}^{\infty}\left(\int_{s}^{\infty} \frac{w(t)}{t^{2 q}} \mathrm{~d} t\right)^{\frac{r_{2}}{p_{2}}} \frac{w(s)}{s^{2 q}} \sup _{y \in(0, s)} y^{r_{2}} V_{2}^{-\frac{r_{2}}{p_{2}}}(y) \mathrm{d} s\right)^{\frac{p_{1}}{p_{1}-r_{2}}}\right. \\
& \left.\times\left(\int_{x}^{\infty} \frac{w(t)}{t^{2 q}} \mathrm{~d} t\right)^{\frac{r_{2}}{p_{2}}} \frac{w(x)}{x^{2 q}} \sup _{y \in(0, x)} y^{r_{2}} V_{2}^{-\frac{r_{2}}{p_{2}}}(y)\left(\int_{0}^{x} \frac{s^{p_{1}^{\prime}} v_{1}(s)}{V_{1}^{p_{1}^{\prime}}(s)} \mathrm{d} s\right)^{\frac{r_{2}\left(p_{1}-1\right)}{p_{1}-r_{2}}} \mathrm{~d} x\right]^{\frac{1}{R}} \\
& +\left[\int_{0}^{\infty}\left(\int_{x}^{\infty}\left(\int_{s}^{\infty} \frac{w(t)}{t^{2 q}} \mathrm{~d} t\right)^{\frac{r_{1}}{p_{1}}} \frac{w(s)}{s^{2 q}}\left(\int_{0}^{s} \frac{y^{p_{1}^{\prime}} v_{1}(y)}{V_{1}^{p_{1}^{\prime}}(y)} \mathrm{d} y\right)^{\frac{r_{1}}{p_{1}^{\prime}}} \mathrm{d} s\right)^{\frac{r_{1}}{p_{2}-r_{1}}}\right. \\
& \left.\times\left(\int_{x}^{\infty} \frac{w(s)}{s^{2 q}} \mathrm{~d} s\right)^{\frac{r_{1}}{p_{1}}} \frac{w(x)}{x^{2 q}}\left(\int_{0}^{x} \frac{s^{p_{1}^{\prime}} v_{1}(s)}{V_{1}^{p_{1}^{\prime}}(s)} \mathrm{d} s\right)^{\frac{r_{1}}{p_{1}^{\prime}}} \sup _{y \in(0, x)} y^{R} V_{2}^{\frac{r_{1}}{r_{1}-p_{2}}}(y) \mathrm{d} x\right]^{\frac{1}{R}} .
\end{aligned}
$$

(v) Let $p_{1}, p_{2} \leq 1$ and $\frac{1}{q} \leq \frac{1}{p_{1}}+\frac{1}{p_{2}}$. Then $C_{(1)} \simeq A_{(61)}^{1,2}+A_{(61)}^{2,1}+A_{(70)}^{1,2}+$ $A_{(70)}^{2,1}+A_{(71)}^{1,2}+A_{(71)}^{2,1}+A_{(72)}^{1,2}+A_{(72)}^{2,1}$, where

(70) $A_{(70)}^{i, j}:=\sup _{x>0}\left[\int_{0}^{x}\left(\int_{s}^{x} \frac{w(t)}{t^{q}} \mathrm{~d} t\right)^{\frac{r_{i}}{p_{i}}} \frac{w(s)}{s^{q}} \sup _{y \in(0, s)} y^{r_{i}} V_{i}^{-\frac{r_{i}}{p_{i}}}(y) \mathrm{d} s\right]^{\frac{1}{r_{i}}} V_{j}^{-\frac{1}{p_{j}}}(x)$, 
(71)

$$
A_{(71)}^{i, j}:=\sup _{x>0} x V_{i}^{-\frac{1}{p_{i}}}(x)\left[\int_{x}^{\infty}\left(\int_{x}^{s} \frac{w(t)}{t^{q}} \mathrm{~d} t\right)^{\frac{r_{j}}{p_{j}}} \frac{w(s)}{s^{q}} V_{j}^{-\frac{r_{j}}{p_{j}}}(s) \mathrm{d} s\right]^{\frac{1}{r_{j}}},
$$

and

(72) $A_{(72)}^{i, j}:=\sup _{x>0} x V_{i}^{-\frac{1}{p_{i}}}(x)\left[\int_{x}^{\infty}\left(\int_{s}^{\infty} \frac{w(t)}{t^{2 q}} \mathrm{~d} t\right)^{\frac{r_{j}}{p_{j}}} \frac{w(s)}{s^{2 q}} \sup _{y \in(0, s)} y^{r_{j}} V_{j}^{-\frac{r_{j}}{p_{j}}}(y) \mathrm{d} s\right]^{\frac{1}{r_{j}}}$.

(vi) Let $p_{1}, p_{2} \leq 1$ and $\frac{1}{q}>\frac{1}{p_{1}}+\frac{1}{p_{2}}$. Then $C_{(1)} \simeq A_{(65)}^{1,2}+A_{(65)}^{2,1}+A_{(73)}^{1,2}+$ $A_{(73)}^{2,1}+A_{(74)}^{1,2}+A_{(74)}^{2,1}+A_{(75)}^{1,2}+A_{(75)}^{2,1}$, where

$$
\begin{aligned}
A_{(73)}^{i, j}:= & {\left[\int_{0}^{\infty}\left(\int_{0}^{x}\left(\int_{s}^{x} \frac{w(t)}{t^{q}} \mathrm{~d} t\right)^{\frac{r_{j}}{p_{j}}} \frac{w(s)}{s^{q}} \sup _{y \in(0, s)} y^{r_{j}} V_{j}^{-\frac{r_{j}}{p_{j}}}(y)\right)^{\frac{r_{j}}{p_{i}-r_{j}}}\right.} \\
& \left.\times \frac{w(x)}{x^{q}} \int_{0}^{x}\left(\int_{s}^{x} \frac{w(t)}{t^{q}} \mathrm{~d} t\right)^{\frac{r_{j}-p_{j}}{p_{j}}} \frac{w(s)}{s^{q}} \sup _{y \in(0, s)} y^{r_{j}} V_{j}^{-\frac{r_{j}}{p_{j}}}(y) \mathrm{d} s V_{i}^{-\frac{r_{i}}{p_{i}}}(x) \mathrm{d} x\right]^{\frac{1}{R}},
\end{aligned}
$$

$$
\begin{aligned}
A_{(74)}^{i, j}:= & {\left[\int_{0}^{\infty}\left(\int_{x}^{\infty}\left(\int_{x}^{s} \frac{w(t)}{t^{q}} \mathrm{~d} t\right)^{\frac{r_{j}}{p_{j}}} \frac{w(s)}{s^{q}} V_{j}^{-\frac{r_{j}}{p_{j}}}(s) \mathrm{d} s\right)^{\frac{r_{j}}{p_{i}-r_{j}}}\right.} \\
& \left.\times \frac{w(x)}{x^{q}} \int_{x}^{\infty}\left(\int_{x}^{s} \frac{w(t)}{t^{q}} \mathrm{~d} t\right)^{\frac{r_{j}-p_{j}}{p_{j}}} \frac{w(s)}{s^{q}} V_{j}^{-\frac{r_{j}}{p_{j}}}(s) \mathrm{d} s \sup _{y \in(0, x)} y^{R} V_{i}^{\frac{r_{j}}{r_{j}-p_{i}}}(y) \mathrm{d} x\right]^{\frac{1}{R}},
\end{aligned}
$$

and

$$
\begin{aligned}
A_{(75)}^{i, j}:= & {\left[\int_{0}^{\infty}\left(\int_{x}^{\infty}\left(\int_{s}^{\infty} \frac{w(t)}{t^{2 q}} \mathrm{~d} t\right)^{\frac{r_{j}}{p_{j}}} \frac{w(s)}{s^{2 q}} \sup _{y \in(0, s)} y^{r_{j}} V_{j}^{-\frac{r_{j}}{p_{j}}}(y) \mathrm{d} s\right)^{\frac{r_{j}}{p_{i}-r_{j}}}\right.} \\
& \left.\times\left(\int_{x}^{\infty} \frac{w(t)}{t^{2 q}} \mathrm{~d} t\right)^{\frac{r_{j}}{p_{j}}} \frac{w(x)}{x^{2 q}} \sup _{y \in(0, x)} y^{r_{j}} V_{j}^{-\frac{r_{j}}{p_{j}}}(y) \sup _{t \in(0, x)} t^{R} V_{i}^{\frac{r_{j}}{r_{j}-p_{i}}}(t) \mathrm{d} x\right]^{\frac{1}{R}} .
\end{aligned}
$$


Proof: Consider first the case $1<p_{1}$. Assume that the function $u_{1}$ defined by

$$
u_{1}(x):=\int_{0}^{x} \frac{s^{p_{1}^{\prime}} v_{1}(s)}{V_{1}^{p_{1}^{\prime}}(s)} \mathrm{d} s, \quad x>0
$$

is integrable near the origin. Then, applying Proposition 2.3, we obtain $(76)$

$$
\begin{aligned}
& C_{(1)} \simeq \sup _{g \in \mathscr{M}} \frac{\left(\int_{0}^{\infty}\left(\int_{0}^{x}\left(g^{* *}\right)^{q} w\right)^{\frac{r_{1}}{q}} V_{1}^{-\frac{r_{1}}{q}}(x) v_{1}(x) \mathrm{d} x\right)^{\frac{1}{r_{1}}}}{\left(\int_{0}^{\infty}\left(g^{*}\right)^{p_{2}} v_{2}\right)^{\frac{1}{p_{2}}}} \\
& \quad+\sup _{g \in \mathscr{M}} \frac{\left(\int_{0}^{\infty}\left(\int_{x}^{\infty} \frac{\left(\left(g^{* *}(s)\right)^{q} w(s)\right.}{s^{q}} \mathrm{~d} s\right)^{\frac{r_{1}}{q}}\left(\int_{0}^{x} \frac{s^{p^{\prime}} v_{1}(s)}{V_{1}^{p_{1}^{\prime}}(s)} \mathrm{d} s\right)^{\frac{r_{1}}{q^{\prime}}} \frac{x^{p_{1}^{\prime}} v_{1}(x)}{V_{1}^{p_{1}^{\prime}}(x)} \mathrm{d} x\right)^{\frac{1}{r_{1}}}}{\left(\int_{0}^{\infty}\left(g^{*}\right)^{p_{2}} v_{2}\right)^{\frac{1}{p_{2}}}} \\
& \quad+\sup _{g \in \mathscr{M}} \frac{\left(\int_{0}^{\infty}\left(g^{* *}\right)^{q} w\right)^{\frac{1}{q}} V_{1}^{-\frac{1}{p_{1}}}(\infty)}{\left(\int_{0}^{\infty}\left(g^{*}\right)^{p_{2}} v_{2}\right)^{\frac{1}{p_{2}}}} \\
&=: B_{1}+B_{2}+B_{3} .
\end{aligned}
$$

(i) We use Lemma 3.2(i) with the setting $\alpha:=q, \beta:=r_{1}, \gamma:=p_{2}$, $\varphi:=w, \psi(t):=V_{1}^{-\frac{r_{1}}{q}}(t) v_{1}(t), \omega:=v_{2}$, we obtain the characterization of $B_{1}$, and Proposition 2.3 to get the characterization of $B_{3}$. We obtain the equivalence

$$
B_{1}+B_{3} \simeq B_{4}+A_{(62)}^{2,1}+A_{(63)}^{2,1},
$$

where

$$
B_{4}:=\sup _{x>0}\left(\int_{0}^{x} W^{\frac{r_{1}}{q}} V_{1}^{-\frac{r_{1}}{q}} v_{1}\right)^{\frac{1}{r_{1}}} V_{2}^{-\frac{1}{p_{2}}}(x)+\sup _{x>0}\left(\int_{0}^{x} W^{\frac{r_{2}}{q}} V_{2}^{-\frac{r_{2}}{q}} v_{2}\right)^{\frac{1}{r_{2}}} V_{1}^{-\frac{1}{p_{1}}}(x) .
$$

Integration by parts yields

$$
A_{(61)}^{1,2}+A_{(61)}^{2,1} \simeq B_{4}+\sup _{x>0} W^{\frac{1}{q}}(x) V_{1}^{-\frac{1}{p_{1}}}(x) V_{2}^{-\frac{1}{p_{2}}}(x) .
$$


Moreover, the following series of inequalities holds true.

$$
\begin{aligned}
\sup _{x>0} & W^{\frac{1}{q}}(x) V_{1}^{-\frac{1}{p_{1}}}(x) V_{2}^{-\frac{1}{p_{2}}}(x) \\
& \simeq \sup _{g \in \mathscr{M}} \frac{\left(\int_{0}^{\infty}\left(g^{*}(t)\right)^{r_{1}} W^{\frac{r_{1}}{p_{1}}}(t) w(t) V_{1}^{-\frac{r_{1}}{p_{j}}}(t) \mathrm{d} t\right)^{\frac{1}{r_{1}}}}{\left(\int_{0}^{\infty}\left(g^{*}(t)\right)^{p_{2}} v_{2}(t) \mathrm{d} t\right)^{\frac{1}{p_{2}}}} \\
& \lesssim \sup _{g \in \mathscr{M}} \frac{\left(\int_{0}^{\infty}\left(g^{* *}(t)\right)^{r_{1}} W^{\frac{r_{1}}{p_{1}}}(t) w(t) V_{1}^{-\frac{r_{1}}{p_{j}}}(t) \mathrm{d} t\right)^{\frac{1}{r_{1}}}}{\left(\int_{0}^{\infty}\left(g^{*}(t)\right)^{p_{2}} v_{2}(t) \mathrm{d} t\right)^{\frac{1}{p_{2}}}} \\
& \leq \sup _{g \in \mathscr{M}} \frac{\left(\int_{0}^{\infty}\left(\int_{0}^{t}\left(g^{* *}\right)^{q} w\right)^{\frac{r_{1}}{p_{1}}}\left(g^{* *}(t)\right)^{q} w(t) V_{1}^{-\frac{r_{1}}{p_{j}}}(t) \mathrm{d} t\right)^{\frac{1}{r_{1}}}}{\left(\int_{0}^{\infty}\left(g^{*}(t)\right)^{p_{2}} v_{2}(t) \mathrm{d} t\right)^{\frac{1}{p_{2}}}} \\
& \simeq B_{1}+B_{3} .
\end{aligned}
$$

The first step is due to the characterization of $\Lambda \hookrightarrow \Lambda[\mathbf{7}$, Theorem 3.1(ii)] and the last equivalence follows by integration by parts. Notice that the resulting relation

$$
\sup _{x>0} W^{\frac{1}{q}}(x) V_{1}^{-\frac{1}{p_{1}}}(x) V_{2}^{-\frac{1}{p_{2}}}(x) \lesssim B_{1}+B_{3}
$$

is established also if we consider the settings of cases (iii) and (v), i.e. if $p_{1} \leq 1$ or $p_{2} \leq 1$ and the other relations between the parameters remain unchanged. To continue, combining the obtained estimates we get

$$
B_{1}+B_{3} \simeq A_{(61)}^{1,2}+A_{(61)}^{2,1}+A_{(62)}^{2,1}+A_{(63)}^{2,1} .
$$

To deal with $B_{2}$, we use Lemma 3.3(i), setting $\alpha:=q, \beta:=r_{1}, \gamma:=p_{2}$, $\varphi(t):=\frac{w(t)}{t^{q}}, \psi(t):=\left(\int_{0}^{t} s^{p_{1}^{\prime}} v_{1}(s) V_{1}^{-p_{1}^{\prime}}(s) \mathrm{d} s\right)^{\frac{r_{1}}{q^{\prime}}} t^{p_{1}^{\prime}} v_{1}(t) V_{1}^{-p_{1}^{\prime}}(t), \omega:=$ 
$v_{2}$. We obtain

$$
\begin{aligned}
& B_{2} \simeq A_{(62)}^{1,2}+A_{(64)}^{1,2} \\
&+\sup _{x>0}\left[\int_{0}^{x}\left(\int_{s}^{x} \frac{w(t)}{t^{q}} \mathrm{~d} t\right)^{\frac{r_{1}}{q}}\left(\int_{0}^{s} \frac{t^{p_{1}^{\prime}} v_{1}(t)}{V_{1}^{p_{1}^{\prime}}(t)} \mathrm{d} t\right)^{\frac{r_{1}}{q^{\prime}}} \frac{s^{p_{1}^{\prime}} v_{1}(s)}{V_{1}^{p_{1}^{\prime}}(s)} \mathrm{d} s\right]^{\frac{1}{r_{1}}} V_{2}^{-\frac{1}{p_{2}}}(x) \\
&+\sup _{x>0}\left[\int_{x}^{\infty}\left(\int_{s}^{\infty} \frac{w(t)}{t^{2 q}} \mathrm{~d} t\right)^{\frac{r_{1}}{q}}\left(\int_{0}^{s} \frac{t^{p_{1}^{\prime}} v_{1}(t)}{V_{1}^{p_{1}^{\prime}}(t)} \mathrm{d} t\right)^{\frac{r_{1}}{q^{\prime}}} \frac{s^{p_{1}^{\prime}} v_{1}(s)}{V_{1}^{p_{1}^{\prime}}(s)} \mathrm{d} s\right]^{\frac{1}{r_{1}}} \\
& \times\left(\int_{0}^{x} \frac{t^{p_{2}^{\prime}} v_{2}(t)}{V_{2}^{p_{2}^{\prime}}(t)} \mathrm{d} t\right)^{\frac{1}{p_{2}^{\prime}}} .
\end{aligned}
$$

We now handle the third term in the sum by integration by parts and the fourth one in the same way as an analogous term in the proof of Lemma 3.3(i), concluding that $B_{2} \simeq A_{(62)}^{1,2}+A_{(63)}^{1,2}+A_{(64)}^{1,2}+A_{(64)}^{2,1}$. Together we get

$$
C_{(1)} \simeq A_{(61)}^{1,2}+A_{(61)}^{2,1}+A_{(62)}^{1,2}+A_{(62)}^{2,1}+A_{(63)}^{1,2}+A_{(63)}^{2,1}+A_{(64)}^{1,2}+A_{(64)}^{2,1},
$$

still assuming the integrability of $u_{1}$ near the origin. Now we perform the usual final argument to drop the assumption on $u_{1}$. If $u_{1}$ is not integrable near the origin, then both $A_{(62)}^{1,2}=\infty$ and $B_{2}=\infty$, the latter by Proposition 2.3. Since $B_{2}=\infty$, it also holds $C_{(1)}=\infty$. Then the both sides of (79) are infinite, hence the equivalence holds trivially. The same argument may be repeated in cases (ii)-(iv), only replacing $A_{(62)}^{1,2}$ with another appropriate condition, when needed.

(ii) Here we use Lemmas 3.2(ii) and 3.3(ii) again, with the same respective settings of parameters as in the case (i), to estimate $B_{1}$ and $B_{2}$. Besides that, we also make use of Proposition 2.3 to estimate $B_{3}$. For $B_{1}$ and $B_{3}$ we so obtain

$$
B_{1}+B_{3} \simeq A_{(65)}^{1,2}+A_{(65)}^{2,1}+A_{(66)}^{2,1} .
$$

In order to get this equivalence, we in fact also need to prove the inequality

$$
\left[\int_{0}^{\infty}\left(\int_{0}^{x} W^{\frac{r_{1}}{p_{1}}} w V_{1}^{-\frac{r_{1}}{p_{1}}}\right)^{\frac{r_{1}}{p_{2}-r_{1}}} W^{\frac{r_{1}}{p_{1}}}(x) w(x) V_{1}^{-\frac{r_{1}}{p_{1}}}(x) V_{2}^{\frac{r_{1}}{r_{1}-p_{2}}}(x) \mathrm{d} x\right]^{\frac{1}{R}} \lesssim B_{1}+B_{3} .
$$

It is done by reusing the argument used to establish (77) (notice the supremal condition from (77) being replaced by an integral condition 
this time, this is due to the different setting of parameters). The above inequality is also true in case (iv). Now we continue with $B_{2}$. We get

$$
\begin{aligned}
B_{2} \simeq & \int_{0}^{\infty}\left(\int_{x}^{\infty}\left(\int_{x}^{s} \frac{w(t)}{t^{q}} \mathrm{~d} t\right)^{\frac{r_{2}}{q}} V_{2}^{-\frac{r_{2}}{q}}(s) v_{2}(s) \mathrm{d} s\right)^{\frac{r_{2}}{p_{1}-r_{2}}} \frac{w(x)}{x^{q}} \\
& \left.\times \int_{x}^{\infty}\left(\int_{x}^{y} \frac{w(t)}{t^{q}} \mathrm{~d} t\right)^{\frac{r_{2}}{p_{2}}} V_{2}^{-\frac{r_{2}}{q}}(y) v_{2}(y) \mathrm{d} y\left(\int_{0}^{x} \frac{s^{p_{1}^{\prime}} v_{1}(s)}{V^{p_{1}^{\prime}}(s)} \mathrm{d} s\right)^{\frac{r_{2}\left(p_{1}-1\right)}{p_{1}-r_{2}}} \mathrm{~d} x\right]^{\frac{1}{R}} \\
+ & {\left[\int_{0}^{\infty}\left(\int_{0}^{x}\left(\int_{s}^{x} \frac{w(t)}{t^{q}} \mathrm{~d} t\right)^{\frac{r_{1}}{q}}\left(\int_{0}^{s} \frac{y^{p_{1}^{\prime}} v_{1}(y)}{V_{1}^{p_{1}^{\prime}}(y)} \mathrm{d} y\right)^{\frac{r_{1}}{q^{\prime}}} \frac{s^{p_{1}^{\prime}} v_{1}(s)}{V_{1}^{p_{1}^{\prime}}(s)} \mathrm{d} s\right)^{\frac{r_{1}}{p_{2}-r_{1}}}\right.} \\
& \left.\times \frac{w(x)}{x^{q}} \int_{0}^{x}\left(\int_{s}^{x} \frac{w(t)}{t^{q}} \mathrm{~d} t\right)^{\frac{r_{1}}{p_{1}}}\left(\int_{0}^{s} \frac{y^{p_{1}^{\prime}} v_{1}(y)}{V_{1}^{p_{1}^{\prime}}(y)} \mathrm{d} y\right)^{\frac{r_{1}}{q^{\prime}}} \frac{s^{p_{1}^{\prime}} v_{1}(s)}{V_{1}^{p_{1}^{\prime}}(s)} \mathrm{d} s V_{2}^{\frac{r_{1}}{r_{1}-p_{2}}}(x) \mathrm{d} x\right]^{\frac{1}{R}} \\
+ & B_{5}+B_{6},
\end{aligned}
$$

where

$$
\begin{aligned}
& B_{5}:=\left[\int_{0}^{\infty}\left(\int_{x}^{\infty}\left(\int_{s}^{\infty} \frac{w(t)}{t^{2 q}} \mathrm{~d} t\right)^{\frac{r_{2}}{p_{2}}} \frac{w(s)}{s^{2 q}}\left(\int_{0}^{s} \frac{y^{p_{2}^{\prime}} v_{2}(y)}{V_{2}^{p_{2}^{\prime}}(y)} \mathrm{d} y\right)^{\frac{r_{2}}{p_{2}^{\prime}}} \mathrm{d} s\right)^{\frac{p_{1}}{p_{1}-r_{2}}}\right. \\
&\left.\times\left(\int_{0}^{x} \frac{s^{p_{1}^{\prime}} v_{1}(s)}{V_{1}^{p_{1}^{\prime}}(s)} \mathrm{d} s\right)^{\frac{p_{1}\left(r_{2}-1\right)}{p_{1}-r_{2}}} \frac{x^{p_{1}^{\prime}} v_{1}(x)}{V_{1}^{p_{1}^{\prime}}(x)} \mathrm{d} x\right]^{\frac{1}{R}}
\end{aligned}
$$

and

$$
\begin{array}{r}
B_{6}:=\left[\int_{0}^{\infty}\left(\int_{x}^{\infty}\left(\int_{s}^{\infty} \frac{w(t)}{t^{2 q}} \mathrm{~d} t\right)^{\frac{r_{1}}{q}}\left(\int_{0}^{s} \frac{y^{p_{1}^{\prime}} v_{1}(y)}{V_{1}^{p_{1}^{\prime}}(y)} \mathrm{d} y\right)^{\frac{r_{1}}{q^{\prime}}} \frac{s^{p_{1}^{\prime}} v_{1}(s)}{V_{1}^{p_{1}^{\prime}}(s)} \mathrm{d} s\right)^{\frac{r_{1}}{p_{2}-r_{1}}}\right. \\
\times\left(\int_{x}^{\infty} \frac{w(t)}{t^{2 q}} \mathrm{~d} t\right)^{\frac{r_{1}}{q}}\left(\int_{0}^{x} \frac{y^{p_{1}^{\prime}} v_{1}(y)}{V_{1}^{p_{1}^{\prime}}(y)} \mathrm{d} y\right)^{\frac{r_{1}}{q^{\prime}}} \frac{x^{p_{1}^{\prime}} v_{1}(x)}{V_{1}^{p_{1}^{\prime}}(x)} \\
\left.\times\left(\int_{0}^{x} \frac{s^{p_{2}^{\prime}} v_{2}(s)}{V_{2}^{p_{2}^{\prime}}(s)} \mathrm{d} s\right)^{\frac{r_{1}\left(p_{2}-1\right)}{p_{2}-r_{1}}} \mathrm{~d} x\right]^{\frac{1}{R}} .
\end{array}
$$

Using integration by parts together with Proposition 2.1, one shows that the first two terms in $B_{2}$ are equivalent to $A_{(66)}^{1,2}$, hence $B_{2} \simeq A_{(66)}^{1,2}+$ 
$B_{5}+B_{6}$. Similarly we prove that $B_{5} \simeq A_{(67)}^{2,1}$. Next, again by integration by parts we get

$$
\begin{array}{r}
A_{(67)}^{1,2} \simeq B_{6}+\left[\int_{0}^{\infty}\left(\int_{x}^{\infty} \frac{w(t)}{t^{2 q}} \mathrm{~d} t\right)^{\frac{r_{1} p_{2}}{q\left(p_{2}-r_{1}\right)}}\left(\int_{0}^{x} \frac{y^{p_{1}^{\prime}} v_{1}(y)}{V_{1}^{p_{1}^{\prime}}(y)} \mathrm{d} y\right)^{\frac{r_{1} p_{2}}{p_{1}^{\prime}\left(p_{2}-r_{1}\right)}}\right. \\
\left.\quad \times\left(\int_{0}^{x} \frac{s^{p_{2}^{\prime}} v_{2}(s)}{V_{2}^{p_{2}^{\prime}}(s)} \mathrm{d} s\right)^{\frac{p_{2}\left(r_{1}-1\right)}{p_{2}-r_{1}}} \mathrm{~d} x\right]^{\frac{1}{R}} \lesssim B_{6}+B_{5},
\end{array}
$$

hence $B_{5}+B_{6} \simeq A_{(67)}^{1,2}+A_{(67)}^{2,1}$ and therefore also $B_{2} \simeq A_{(66)}^{1,2}+A_{(67)}^{1,2}+$ $A_{(67)}^{2,1}$. Altogether, it holds

$$
C_{(1)} \simeq B_{1}+B_{2}+B_{3} \simeq A_{(65)}^{1,2}+A_{(65)}^{2,1}+A_{(66)}^{1,2}+A_{(66)}^{2,1}+A_{(67)}^{1,2}+A_{(67)}^{2,1} .
$$

Finally, the assumption of integrability of $u_{1}$ is removed in a similar way as in (i).

(iii) Using Lemmas 3.2(iii) and 3.3(iii) with the same setting as in (i) and then repeating the argument from (i) to show (78), we get

$$
C_{(1)} \simeq B_{1}+B_{2}+B_{3} \simeq A_{(61)}^{1,2}+A_{(61)}^{2,1}+A_{(62)}^{1,2}+A_{(63)}^{1,2}+A_{(68)} .
$$

Then we prove that this statement holds also if $u_{1}$ is not integrable near the origin, by imitating the argument from (i).

(iv) Here we use Lemmas 3.2(iv) and 3.3(iv) to get the estimate of $B_{1}+B_{2}+B_{3}$. Further adjustments of the conditions are made using the corresponding arguments from (ii). We omit the details.

Now suppose that $p_{1} \leq 1$, which is the case in (v) and (vi). For $i \in\{1,2\}$ denote

$$
\sigma_{i}(x):=\sup _{0<y \leq x} y^{r_{i}} V_{i}^{-\frac{r_{i}}{p_{i}}}(y), \quad x>0 .
$$


Using [6, Theorem 3.1] and integration by parts, we obtain (80)

$$
\begin{aligned}
C_{(1)} \simeq & B_{1}+B_{3} \\
& +\sup _{g \in \mathscr{M}} \frac{\left(\int_{0}^{\infty}\left(\int_{x}^{\infty} \frac{\left(g^{* *}(t)\right)^{q} w(t)}{t^{q}} \mathrm{~d} t\right)^{\frac{r_{1}}{p_{1}}} \frac{\left(g^{* *}(x)\right)^{q} w(x)}{x^{q}} \sigma_{1}(x) \mathrm{d} x\right)^{\frac{1}{r_{1}}}}{\left(\int_{0}^{\infty}\left(g^{*}\right)^{p_{2}} v_{2}\right)^{\frac{1}{p_{2}}}} \\
\simeq & B_{1}+B_{3}+\sup _{g \in \mathscr{M}} \frac{\left(\int_{0}^{\infty}\left(\int_{x}^{\infty} \frac{\left(g^{* *}(t)\right)^{q} w(t)}{t^{q}} \mathrm{~d} t\right)^{\frac{r_{1}}{q}} \sigma_{1}^{\prime}(x) \mathrm{d} x\right)^{\frac{1}{r_{1}}}}{\left(\int_{0}^{\infty}\left(g^{*}\right)^{p_{2}} v_{2}\right)^{\frac{1}{p_{2}}}} \\
& +\sup _{g \in \mathscr{M}} \frac{\sigma_{1}^{\frac{1}{r_{1}}}(0+)\left(\int_{0}^{\infty} \frac{\left(g^{* *}(x)\right)^{q} w(x)}{x^{q}} \mathrm{~d} x\right)^{\frac{1}{q}}}{\left(\int_{0}^{\infty}\left(g^{*}\right)^{p_{2}} v_{2}\right)^{\frac{1}{p_{2}}}} \\
= & B_{1}+B_{3}+B_{7}+B_{8} .
\end{aligned}
$$

(v) We use Lemma 3.2(iii), setting $\alpha:=q, \beta:=r_{1}, \gamma:=p_{2}, \varphi:=$ $w, \psi:=V_{1}^{-\frac{r_{1}}{q}} v_{1}, \omega:=v_{2}$, to obtain estimates of $B_{1}$; Lemma 3.3(iii), setting $\alpha:=q, \beta:=r_{1}, \gamma:=p_{2}, \varphi(t):=\frac{w(t)}{t^{q}}, \psi:=\sigma_{1}^{\prime}, \omega:=v_{2}$, to estimate $B_{7}$; and [6, Theorem 3.1] to estimate $B_{3}$ and $B_{8}$. Using the obtained expressions in (80) and applying also the argument used in (i) to show (77), we get

$$
C_{(1)} \simeq A_{(61)}^{1,2}+A_{(61)}^{2,1}+B_{9}+B_{10}+B_{11}+B_{12}+B_{13}+A_{(70)}^{2,1}+A_{(72)}^{1,2},
$$

where

$$
\begin{aligned}
B_{9} & :=\sup _{x>0} \sigma_{2}^{\frac{1}{r_{2}}}(x)\left[\int_{x}^{\infty}\left(\int_{x}^{s} \frac{w(t)}{t^{q}} \mathrm{~d} t\right)^{\frac{r_{1}}{q}} V_{1}^{-\frac{r_{1}}{q}}(s) v_{1}(s) \mathrm{d} s\right]^{\frac{1}{r_{1}}}, \\
B_{10} & :=\sigma_{1}^{\frac{1}{r_{1}}}(0+)\left[\int_{0}^{\infty}\left(\int_{0}^{x} \frac{w(t)}{t^{q}} \mathrm{~d} t\right)^{\frac{r_{2}}{p_{2}}} \frac{w(x)}{x^{q}} V_{2}^{-\frac{r_{2}}{p_{2}}}(x) \mathrm{d} x\right]^{\frac{1}{r_{2}}}, \\
B_{11} & :=\sup _{x>0}\left(\int_{0}^{x} \sigma_{1}^{\prime}\right)^{\frac{1}{r_{1}}}\left[\int_{x}^{\infty}\left(\int_{x}^{s} \frac{w(t)}{t^{q}} \mathrm{~d} t\right)^{\frac{r_{2}}{q}} V_{2}^{-\frac{r_{2}}{q}}(s) v_{2}(s) \mathrm{d} s\right]^{\frac{1}{r_{2}}}, \\
B_{12} & :=\sup _{x>0}\left[\int_{0}^{x}\left(\int_{s}^{x} \frac{w(t)}{t^{q}} \mathrm{~d} t\right)^{\frac{r_{1}}{q}} \sigma_{1}^{\prime}(s) \mathrm{d} s\right]^{\frac{1}{r_{1}}} V_{2}^{-\frac{1}{p_{2}}}(x), \\
B_{13} & :=\sup _{x>0} \sigma_{2}^{\frac{1}{r_{2}}}(x)\left[\int_{x}^{\infty}\left(\int_{s}^{\infty} \frac{w(t)}{t^{2 q}} \mathrm{~d} t\right)^{\frac{r_{1}}{q}} \sigma_{1}^{\prime}(s) \mathrm{d} s\right]^{\frac{1}{r_{1}}} .
\end{aligned}
$$


By integration by parts one verifies the following inequalities: $B_{9} \lesssim$ $A_{(71)}^{2,1}, B_{10}+B_{11} \lesssim A_{(71)}^{1,2}, B_{12} \lesssim A_{(70)}^{1,2}$, and $B_{13} \lesssim A_{(72)}^{2,1}$. From these estimates and (81) it follows

$$
C_{(1)} \lesssim A_{(61)}^{1,2}+A_{(61)}^{2,1}+A_{(70)}^{1,2}+A_{(70)}^{2,1}+A_{(71)}^{1,2}+A_{(71)}^{2,1}+A_{(72)}^{1,2}+A_{(72)}^{2,1} .
$$

Next, integration by parts yields the following: $A_{(70)}^{1,2} \lesssim B_{10}+B_{12}$, $A_{(71)}^{1,2} \lesssim B_{10}+B_{11}+B_{12}, A_{(71)}^{2,1} \lesssim B_{9}+A_{(71)}^{2,1}$, and $A_{(72)}^{2,1} \lesssim B_{13}+A_{(72)}^{1,2}$. Using all these inequalities in (81), we get

$$
A_{(61)}^{1,2}+A_{(61)}^{2,1}+A_{(70)}^{1,2}+A_{(70)}^{2,1}+A_{(71)}^{1,2}+A_{(71)}^{2,1}+A_{(72)}^{1,2}+A_{(72)}^{2,1} \lesssim C_{(1)} .
$$

The proof of this part is then completed.

(vi) Analogously to the case (v) we use Lemma 3.2(iv) to estimate $B_{1}$, Lemma 3.3(iv) to estimate $B_{7}$, and [6, Theorem 3.1] to get an estimate of $B_{3}$ and $B_{8}$. Inserting these expressions into (80) and merging some of them by integration by parts (similarly to the case (ii)), we obtain

$$
C_{(1)} \simeq A_{(65)}^{1,2}+A_{(65)}^{2,1}+A_{(73)}^{1,2}+A_{(75)}^{1,2}+A_{(75)}^{1,2}+B_{10}+B_{14}+B_{15}+B_{16}
$$

where

$$
\begin{aligned}
B_{14}:= & {\left[\int_{0}^{\infty}\left(\int_{x}^{\infty}\left(\int_{x}^{s} \frac{w(t)}{t^{q}} \mathrm{~d} t\right)^{\frac{r_{1}}{q}} V_{1}^{-\frac{r_{1}}{q}}(s) v_{1}(s) \mathrm{d} s\right)^{\frac{r_{1}}{p_{2}-r_{1}}}\right.} \\
& \left.\times \frac{w(x)}{x^{q}} \int_{x}^{\infty}\left(\int_{x}^{s} \frac{w(t)}{t^{q}} \mathrm{~d} t\right)^{\frac{r_{1}}{p_{1}}} V_{1}^{-\frac{r_{1}}{q}}(s) v_{1}(s) \mathrm{d} s \sigma_{2}^{\frac{p_{1}}{p_{1}-r_{2}}}(x) \mathrm{d} x\right]^{\frac{1}{R}}, \\
B_{15}:= & {\left[\int_{0}^{\infty}\left(\int_{x}^{\infty}\left(\int_{x}^{s} \frac{w(t)}{t^{q}} \mathrm{~d} t\right)^{\frac{r_{2}}{q}} V_{2}^{-\frac{r_{2}}{q}}(s) v_{2}(s) \mathrm{d} s\right)^{\frac{p_{1}}{p_{1}-r_{2}}}\left(\int_{0}^{x} \sigma_{1}^{\prime}\right)^{\frac{r_{1}}{p_{2}-r_{1}}} \sigma_{1}^{\prime}(x) \mathrm{d} x\right]^{\frac{1}{R}}, } \\
B_{16}:= & {\left[\int_{0}^{\infty}\left(\int_{0}^{x}\left(\int_{s}^{x} \frac{w(t)}{t^{q}} \mathrm{~d} t\right)^{\frac{r_{1}}{q}} \sigma_{1}^{\prime}(s) \mathrm{d} s\right)^{\frac{r_{1}}{p_{2}-r_{1}}}\right.} \\
& \left.\times \frac{w(x)}{x^{q}} \int_{0}^{x}\left(\int_{s}^{x} \frac{w(t)}{t^{q}} \mathrm{~d} t\right)^{\frac{r_{1}}{p_{1}}} \sigma_{1}^{\prime}(s) \mathrm{d} s V_{2}^{\frac{r_{1}}{r_{1}-p_{2}}}(x) \mathrm{d} x\right]^{\frac{1}{R}} .
\end{aligned}
$$

Performing integration by parts, one gets $B_{10} \lesssim A_{(74)}^{2,1}, B_{14} \lesssim A_{(74)}^{2,1}$, $B_{15} \lesssim A_{(74)}^{1,2}$, and $B_{16} \lesssim A_{(73)}^{1,2}$. We apply these inequalities to replace the " $B$-parts" in (82), and so we obtain

$$
C_{(1)} \lesssim A_{(65)}^{1,2}+A_{(65)}^{2,1}+A_{(73)}^{1,2}+A_{(73)}^{2,1}+A_{(74)}^{1,2}+A_{(74)}^{2,1}+A_{(75)}^{1,2}+A_{(75)}^{2,1} .
$$


Now observe that

$$
\begin{aligned}
(83) A_{(73)}^{2,1} \simeq & B_{16}+\sigma_{1}^{\frac{1}{r_{1}}}(0+)\left(\int_{0}^{\infty} \frac{w(t)}{t^{q}} \mathrm{~d} t\right)^{\frac{1}{q}} V_{2}^{-\frac{1}{p_{2}}}(\infty) \\
& +\sigma_{1}^{\frac{1}{r_{1}}}(0+)\left[\int_{0}^{\infty}\left(\int_{0}^{x} \frac{w(t)}{t^{q}} \mathrm{~d} t\right)^{\frac{R}{q}} V_{2}^{\frac{p_{2}}{r_{1}-p_{2}}}(x) v_{2}(x) \mathrm{d} x\right]^{\frac{1}{R}} \\
\lesssim & B_{16}+B_{10}+\sigma_{1}^{\frac{1}{r_{1}}}(0+)\left[\int_{0}^{\infty}\left(\int_{0}^{x} \frac{w(t)}{t^{q}} \mathrm{~d} t\right)^{\frac{R}{q}} V_{2}^{\frac{p_{2}}{r_{1}-p_{2}}}(x) v_{2}(x) \mathrm{d} x\right]^{\frac{1}{R}} \\
\lesssim & B_{16}+B_{10} \\
(84) \quad & +\sigma_{1}^{\frac{1}{r_{1}}}(0+)\left[\int_{0}^{\infty}\left(\int_{0}^{x} \frac{w(t)}{t^{q}} \mathrm{~d} t\right)^{\frac{r_{2}}{q}}\left(\int_{x}^{\infty} V_{2}^{\frac{p_{2}}{r_{1}-p_{2}}} v_{2}\right)^{-\frac{r_{2}}{p_{1}}} V_{2}^{\frac{p_{2}}{r_{1}-p_{2}}}(x) v_{2}(x) \mathrm{d} x\right]^{\frac{1}{R}} \\
\lesssim & B_{16}+B_{10} .
\end{aligned}
$$

Indeed, the estimates (83) and (85) follow by integration by parts, while (84) is granted by Proposition 2.2. We proved that $A_{(73)}^{2,1} \lesssim B_{16}+B_{10}$. By similar means it is shown that $A_{(74)}^{1,2} \lesssim B_{10}+B_{15}+B_{16}$ and $A_{(74)}^{2,1} \lesssim$ $B_{14}+A_{(73)}^{1,2}$. Using these three estimates together with (82), we get

$$
A_{(65)}^{1,2}+A_{(65)}^{2,1}+A_{(73)}^{1,2}+A_{(73)}^{2,1}+A_{(74)}^{1,2}+A_{(74)}^{2,1}+A_{(75)}^{1,2}+A_{(75)}^{2,1} \lesssim C_{(1)} .
$$

This completes case (vi) and thus the whole proof.

The next part deals with the "weak cases", i.e. such configurations of $p_{1}, p_{2}, q$ that at least one of these exponents is infinite. The following theorem covers the case $q=\infty$.

Theorem 4.4. Let $v_{1}, v_{2}, w$ be weights. Let $q=\infty$.

(i) Let $0<p_{1}, p_{2} \leq 1$. Then $C_{(1)} \simeq A_{(86)}$, where

$$
A_{(86)}:=\underset{x>0}{\operatorname{ess} \sup } \frac{w(x)}{x^{2}} \sup _{s \in(0, x)} s V_{1}^{-\frac{1}{p_{1}}}(s) \sup _{t \in(0, x)} t V_{2}^{-\frac{1}{p_{2}}}(t) \text {. }
$$

(ii) Let $0<p_{1} \leq 1<p_{2}<\infty$. Then $C_{(1)} \simeq A_{(87)}$, where

$$
A_{(87)}:=\underset{x>0}{\operatorname{esssup}} \frac{w(x)}{x^{2}} \sup _{s \in(0, x)} s V_{1}^{-\frac{1}{p_{1}}}(s)\left(\int_{0}^{x} t^{p_{2}^{\prime}-1} V_{2}^{1-p_{2}^{\prime}}(t) \mathrm{d} t\right)^{\frac{1}{p_{2}^{\prime}}} .
$$


(iii) Let $0<p_{1} \leq 1<p_{2}=\infty$. Then $C_{(1)} \simeq A_{(88)}$, where

$$
A_{(88)}:=\operatorname{esssup}_{x>0} \frac{w(x)}{x^{2}} \sup _{s \in(0, x)} s V_{1}^{-\frac{1}{p_{1}}}(s) \int_{0}^{x} \frac{\mathrm{d} t}{\operatorname{ess} \sup _{y \in(0, t)} v_{2}(y)} .
$$

(iv) Let $1<p_{1}, p_{2}<\infty$. Then $C_{(1)} \simeq A_{(89)}$, where

(89) $A_{(89)}:=\underset{x>0}{\operatorname{ess} \sup } \frac{w(x)}{x^{2}}\left(\int_{0}^{x} s^{p_{1}^{\prime}-1} V_{1}^{1-p_{1}^{\prime}}(s) \mathrm{d} s\right)^{\frac{1}{p_{1}^{\prime}}}\left(\int_{0}^{x} t^{p_{2}^{\prime}-1} V_{2}^{1-p_{2}^{\prime}}(t) \mathrm{d} t\right)^{\frac{1}{p_{2}^{\prime}}}$.

(v) Let $1<p_{1}<p_{2}=\infty$. Then $C_{(1)} \simeq A_{(90)}$, where

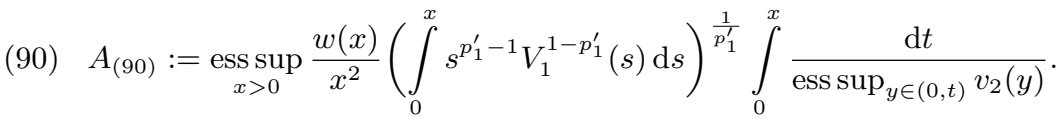

(vi) Let $p_{1}=p_{2}=\infty$. Then $C_{(1)}=A_{(91)}$, where

$$
A_{(91)}:=\operatorname{esssup}_{x>0} \frac{w(x)}{x^{2}} \int_{0}^{x} \frac{\mathrm{d} s}{\operatorname{ess~sup}_{y \in(0, s)} v_{1}(y)} \int_{0}^{x} \frac{\mathrm{d} t}{\operatorname{ess~sup}_{y \in(0, t)} v_{2}(y)} .
$$

Proof: We have

$$
\begin{aligned}
C_{(1)} & =\sup _{f \in \mathscr{M}} \sup _{g \in \mathscr{M}} \frac{\operatorname{ess}^{\sup _{x>0} f^{* *}(x) g^{* *}(x) w(x)}}{\|f\|_{\Lambda^{p_{1}}\left(v_{1}\right)}\|g\|_{\Lambda^{p_{2}\left(v_{2}\right)}}} \\
& =\operatorname{esssup}_{x>0} \frac{w(x)}{x^{2}} \sup _{f \in \mathscr{M}} \frac{\int_{0}^{x} f^{*}(t) \mathrm{d} t}{\|f\|_{\Lambda^{p_{1}}\left(v_{1}\right)}} \sup _{g \in \mathscr{M}} \frac{\int_{0}^{x} g^{*}(t) \mathrm{d} t}{\|g\|_{\Lambda^{p_{2}\left(v_{2}\right)}}} \\
& =\operatorname{ess~sup}_{x>0} \frac{w(x)}{x^{2}}\|\operatorname{Id}\|_{\Lambda^{p_{1}}\left(v_{1}\right) \rightarrow \Lambda^{1}\left(\chi_{(0, x)}\right)}\|\operatorname{Id}\|_{\Lambda^{p_{2}}\left(v_{2}\right) \rightarrow \Lambda^{1}\left(\chi_{(0, x)}\right)} .
\end{aligned}
$$

Now, in all the cases we simply use the characterizations of the embed$\operatorname{ding} \Lambda^{p}(v) \hookrightarrow \Lambda^{1}\left(\chi_{(0, x)}\right)$ provided by [7, Theorem 3.1] and Proposition 2.4 .

Finally, we complete the list with the last remaining case in which $0<q<\infty$ and $0<p_{2} \leq p_{1}=\infty$.

Theorem 4.5. Let $v_{1}, v_{2}, w$ be weights. Let $p_{1}=\infty$ and $0<q<\infty$.

(i) Let $1<p_{2} \leq q$. Then $C_{(1)} \simeq A_{(92)}+A_{(93)}$, where

$$
A_{(92)}:=\sup _{x>0}\left[\int_{0}^{x} \frac{w(s)}{s^{q}}\left(\int_{0}^{s} \frac{\mathrm{d} t}{\operatorname{ess} \sup _{y \in(0, t)} v_{1}(y)}\right)^{q} \mathrm{~d} t\right]^{\frac{1}{q}} V_{2}^{-\frac{1}{p_{2}}}(x)
$$


and

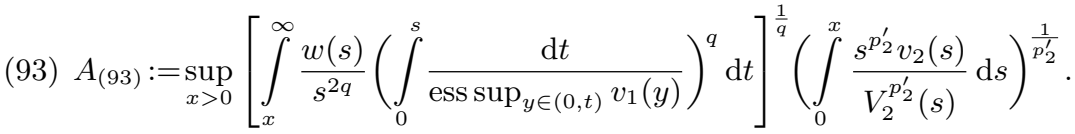

(ii) Let $0<p_{2} \leq 1$ and $p_{2} \leq q$. Then $C_{(1)} \simeq A_{(92)}+A_{(94)}$, where

$$
A_{(94)}:=\sup _{x>0}\left[\int_{x}^{\infty} \frac{w(s)}{s^{2 q}}\left(\int_{0}^{s} \frac{\mathrm{d} t}{\operatorname{ess~sup}_{y \in(0, t)} v_{1}(y)}\right)^{q} \mathrm{~d} t\right]^{\frac{1}{q}} x V_{2}^{-\frac{1}{p_{2}}}(x) .
$$

(iii) Let $1<p_{2}<\infty$ and $0<q<p_{2}$. Then $C_{(1)} \simeq A_{(95)}+A_{(96)}$, where

$$
\begin{aligned}
A_{(95)}:= & {\left[\int_{0}^{\infty}\left(\int_{0}^{x} \frac{w(s)}{s^{q}}\left(\int_{0}^{s} \frac{\mathrm{d} t}{\operatorname{ess~sup}_{y \in(0, t)} v_{1}(y)}\right)^{q} \mathrm{~d} t\right)^{\frac{r_{2}}{p_{2}}}\right.} \\
& \left.\times \frac{w(x)}{x^{q}}\left(\int_{0}^{x} \frac{\mathrm{d} t}{\operatorname{ess~sup}_{y \in(0, t)} v_{1}(y)}\right)^{q} V_{2}^{-\frac{r_{2}}{p_{2}}}(x) \mathrm{d} x\right]^{\frac{1}{r_{2}}}
\end{aligned}
$$

and

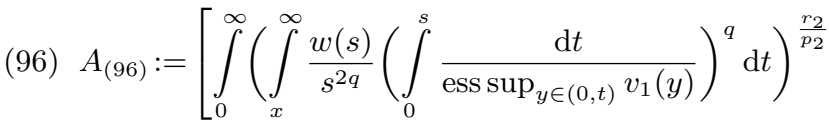

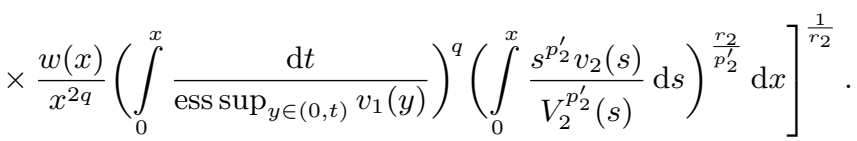

(iv) Let $0<q<p_{2} \leq 1$. Then $C_{(1)} \simeq A_{(95)}+A_{(97)}$, where

$$
\begin{aligned}
& \text { (97) } A_{(97)}:=\left[\int_{0}^{\infty}\left(\int_{x}^{\infty} \frac{w(s)}{s^{2 q}}\left(\int_{0}^{s} \frac{\mathrm{d} t}{\operatorname{ess~sup}_{y \in(0, t)} v_{1}(y)}\right)^{q} \mathrm{~d} t\right)^{\frac{r_{2}}{p_{2}}}\right.
\end{aligned}
$$

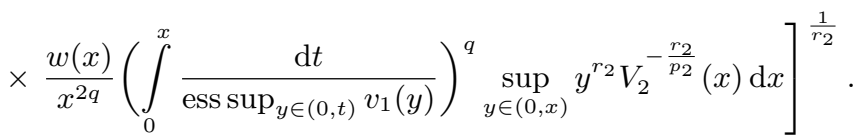

(v) Let $0<q<p_{2}=\infty$. Then $C_{(1)} \simeq A_{(98)}$, where

(98) $A_{(98)}:=\left[\int_{0}^{\infty} \frac{w(x)}{x^{2 q}}\left(\int_{0}^{x} \frac{\mathrm{d} t}{\operatorname{ess~sup}_{y \in(0, t)} v_{1}(y)} \int_{0}^{x} \frac{\mathrm{d} s}{\operatorname{ess~sup}_{y \in(0, s)} v_{2}(y)}\right)^{q} \mathrm{~d} x\right]^{\frac{1}{q}}$. 
Proof: From Proposition 2.4 it follows

$$
\begin{aligned}
& C_{(1)}=\sup _{g \in \mathscr{M}} \sup _{f \in \mathscr{M}} \frac{\left(\int_{0}^{\infty}\left(f^{* *}(x)\right)^{q}\left(g^{* *}(x)\right)^{q} w(x) \mathrm{d} x\right)^{\frac{1}{q}}}{\|f\|_{\Lambda \infty\left(v_{1}\right)}\|g\|_{\Lambda^{p_{2}}\left(v_{2}\right)}} \\
& \simeq \sup _{g \in \mathscr{M}} \frac{\left[\int_{0}^{\infty}\left(g^{* *}(x)\right)^{q} \frac{w(x)}{x^{q}}\left(\int_{0}^{x} \frac{\mathrm{d} s}{\operatorname{ess} \sup _{y \in(0, s)} v_{1}(y)}\right)^{q} \mathrm{~d} x\right]^{\frac{1}{q}}}{\|g\|_{\Lambda^{p_{2}}\left(v_{2}\right)}} \\
& \simeq\|\operatorname{Id}\|_{\Lambda^{p_{2}}\left(v_{2}\right) \rightarrow \Gamma^{q}}\left(x \mapsto \frac { w ( x ) } { x ^ { q } } \left[\left(\operatorname{ess}_{\left.\left.\left.\sup _{y \in(0, s)} v_{1}(y)\right)^{-1}\right]^{q}\right) .}\right.\right.\right.
\end{aligned}
$$

The rest is done by application of the characterization of the involved embedding $\Gamma \hookrightarrow \Lambda$, which can be found in [7, Theorem 4.1] (cases (i) and (ii)), Proposition 2.3 (for case (iii)), [6, Theorem 3.1] (case (iv)), and finally Proposition 2.4 for case (v).

\section{Acknowledgement}

The author would like to thank the referees whose remarks helped to correct and improve the final version of the paper.

\section{References}

[1] M. I. Aguilar Cañestro, P. Ortega Salvador, and C. RaMírez TorreblancA, Weighted bilinear Hardy inequalities, $J$. Math. Anal. Appl. 387(1) (2012), 320-334. DOI: 10.1016/j.jmaa. 2011.08.078.

[2] M. A. Ariño And B. Muckenhoupt, Maximal functions on classical Lorentz spaces and Hardy's inequality with weights for nonincreasing functions, Trans. Amer. Math. Soc. 320(2) (1990), 727-735. DOI: $10.2307 / 2001699$.

[3] C. Bennett and R. Sharpley, "Interpolation of Operators", Pure and Applied Mathematics 129, Academic Press, Inc., Boston, MA, 1988.

[4] D. W. Boyd, The Hilbert transform on rearrangement-invariant spaces, Canad. J. Math. 19 (1967), 599-616. DOI: 10.4153/CJM1967-053-7. 
[5] J. S. Bradley, Hardy inequalities with mixed norms, Canad. Math. Bull. 21(4) (1978), 405-408. DOI: 10.4153/CMB-1978-071-7.

[6] M. Carro, A. Gogatishvili, J. Martín, and L. Pick, Weighted inequalities involving two Hardy operators with applications to embeddings of function spaces, J. Operator Theory 59(2) (2008), 309-332.

[7] M. Carro, L. Pick, J. Soria, and V. D. Stepanov, On embeddings between classical Lorentz spaces, Math. Inequal. Appl. 4(3) (2001), 397-428. DOI: 10.7153/mia-04-37.

[8] M. J. Carro and J. Soria, Weighted Lorentz spaces and the Hardy operator, J. Funct. Anal. 112(2) (1993), 480-494. DOI: 10.1006/jfan.1993.1042.

[9] M. J. CARro And J. Soria, Boundedness of some integral operators, Canad. J. Math. 45(6) (1993), 1155-1166. DOI: 10.4153/CJM1993-064-2.

[10] M. J. Carro and J. Soria, The Hardy-Littlewood maximal function and weighted Lorentz spaces, J. London Math. Soc. (2) 55(1) (1997), 146-158. DOI : 10.1112/S0024610796004462.

[11] A. Gogatishvili, M. Johansson, C. A. Okpoti, and L.-E. Persson, Characterisation of embeddings in Lorentz spaces, Bull. Austral. Math. Soc. 76(1) (2007), 69-92. DOI: 10.1017/S000 4972700039484.

[12] A. Gogatishvili and R. Kerman, The rearrangement-invariant space $\Gamma_{p, \phi}$, Positivity 18(2) (2014), 319-345. DOI: 10.1007/s11117013-0246-4.

[13] A. Gogatishvili, A. Kufner, and L.-E. Persson, Some new scales of weight characterizations of the class $B_{p}$, Acta Math. Hungar. 123(4) (2009), 365-377. DOI: 10.1007/s10474-009-8132-z.

[14] A. Gogatishvili, L.-E. Persson, V. D. Stepanov, and P. WALL, Some scales of equivalent conditions to characterize the Stieltjes inequality: the case $q<p$, Math. Nachr. 287(2-3) (2014), 242-253. DOI: 10.1002/mana. 201200118.

[15] A. Gogatishvili And L. Pick, Discretization and anti-discretization of rearrangement-invariant norms, Publ. Mat. 47(2) (2003), 311-358. DOI : 10.5565/PUBLMAT_47203_02.

[16] A. Gogatishvili, L. Pick, And F. Soudský, Characterization of associate spaces of weighted Lorentz spaces with applications, Studia Math. 224(1) (2014), 1-23. DOI: 10.4064/sm224-1-1. 
[17] A. Gogatishvili and V. D. Stepanov, Reduction theorems for operators on the cones of monotone functions, J. Math. Anal. Appl. 405(1) (2013), 156-172. DOI: 10.1016/j.jmaa.2013.03.046.

[18] M. Ǩ̌EPela, Convolution inequalities in weighted Lorentz spaces, Math. Inequal. Appl. 17(4) (2014), 1201-1223. DOI: 10.7153/mia17-90.

[19] M. KřEPELA, Iterating bilinear Hardy inequalities, Preprint (2015), Proc. Edinb. Math. Soc. (2) (to appear).

[20] A. Kufner, L. Maligranda, and L.-E. Persson, "The Hardy Inequality. About its History and Some Related Results", Vydavatelský Servis, Plzeñ, 2007.

[21] A. Kufner And L.-E. Persson, "Weighted Inequalities of Hardy Type”, World Scientific Publishing Co., Inc., River Edge, NJ, 2003. DOI: $10.1142 / 5129$.

[22] V. G. MAZ'JA, "Sobolev Spaces", Translated from the Russian by T. O. Shaposhnikova, Springer Series in Soviet Mathematics, Springer-Verlag, Berlin, 1985. DOI : 10.1007/978-3-662-09922-3.

[23] B. Muckenhoupt, Hardy's inequality with weights, Collection of articles honoring the completion by Antoni Zygmund of 50 years of scientific activity, I, Studia Math. 44 (1972), 31-38.

[24] R. OǏnarov, Two-sided estimates for the norm of some classes of integral operators, (Russian), Trudy Mat. Inst. Steklov. 204 (1993), 240-250; translation in: Proc. Steklov Inst. Math. 204(3) (1994), 205-214.

[25] B. OpIC And A. Kufner, "Hardy-type Inequalities", Pitman Research Notes in Mathematics Series 219, Longman Scientific \& Technical, Harlow, 1990.

[26] E. SAWYER, Boundedness of classical operators on classical Lorentz spaces, Studia Math. 96(2) (1990), 145-158.

[27] G. Sinnamon, Embeddings of concave functions and duals of Lorentz spaces, Publ. Mat. 46(2) (2002), 489-515. DOI: 10.5565/ PUBLMAT_46202_ 10.

[28] G. Sinnamon and V. D. Stepanov, The weighted Hardy inequality: new proofs and the case $p=1$, J. London Math. Soc. (2) 54(1) (1996), 89-101. DOI: 10.1112/jlms/54.1.89.

[29] J. SoriA, Lorentz spaces of weak-type, Quart. J. Math. Oxford Ser. (2) 49(193) (1998), 93-103.

[30] V. D. Stepanov, The weighted Hardy's inequality for nonincreasing functions, Trans. Amer. Math. Soc. 338(1) (1993), 173-186. DOI: $10.2307 / 2154450$. 
Karlstad University

Faculty of Health, Science and Technology

Department of Mathematics and Computer Science

65188 Karlstad

Sweden

Charles University

Faculty of Mathematics and Physics

Department of Mathematical Analysis

Sokolovská 83

18675 Praha 8

Czech Republic

E-mail address: martin.krepela@kau.se

Primera versió rebuda el 14 de gener de 2015, darrera versió rebuda el 9 de setembre de 2015. 